\title{
Postoperative Multimodal Pain Management and Opioid Consumption in Arthroscopy Clinical Trials: A Systematic Review
}

\author{
Ryan W. Paul, B.S., Patrick F. Szukics, D.O., Joseph Brutico, B.S., \\ Fotios P. Tjoumakaris, M.D., and Kevin B. Freedman, M.D.
}

\begin{abstract}
Purpose: To provide an updated review of multimodal pain management in arthroscopic surgery by evaluating pain and opioid consumption after shoulder, knee, and hip arthroscopy. Methods: A comprehensive literature search was performed to identify randomized controlled trials (RCTs) investigating multimodal pain management after shoulder, knee, and hip arthroscopy. Articles were identified from January 2011 through December 2020 using various databases. As the primary outcome variables of this study, differences in postoperative pain and opioid consumption volumes were summarized from all reported postoperative time points. Results: 37 shoulder, 28 knee, and 8 hip arthroscopy RCTs were included in the study. The most frequent bias present in the included RCTs was incomplete outcome data $(58 \%)$, while group allocation concealment was the least frequent bias (15\%). Qualitative analysis of rotator cuff repair $(n=12)$, anterior cruciate ligament reconstruction $(n=11)$, meniscectomy $(n=5)$, femoroacetabular impingement $(n=2)$, oral medications $(n=8)$, postoperative interventions $(n=10)$, and nonpharmacological interventions $(n=6)$ was performed. Conclusions: Many multimodal pain management protocols offer improved pain control and decreased opioid consumption after arthroscopic surgery. On the basis of the current literature, the evidence supports an interscalene nerve block with a dexamethasone-dexmedetomidine combination for rotator cuff repair, a proximal continuous adductor canal block for anterior cruciate ligament reconstruction, and local infiltration analgesia (e.g., periacetabular injection with 20 $\mathrm{mL}$ of .5\% bupivacaine) for hip arthroscopy. When evaluating oral medication, the evidence supports $150 \mathrm{mg}$ Pregabalin for shoulder arthroscopy, $400 \mathrm{mg}$ Celecoxib for knee arthroscopy, and $200 \mathrm{mg}$ Celecoxib for hip arthroscopy, all taken preoperatively. There is promising evidence for the use of various nonpharmacological modalities, specifically preoperative opioid education for rotator cuff repair patients; however, more clinical trials that evaluate nonpharmacological interventions should be performed. Level of Evidence: Level II, systematic review of Level I and II studies.
\end{abstract}

Rothman Orthopaedic Institute, Philadelphia, Pennsylvania, U.S.A. (R.W.P., J.B., K.B.F.); Rowan University School of Osteopathic Medicine, Department of Orthopaedic Surgery, Stratford, New Jersey, U.S.A. (P.F.S.); and Rothman Orthopaedic Institute, Egg Harbor Township, New Jersey, U.S.A. (F.B.T.).

The authors report the following potential conflicts of interest or sources of funding: K.B. F. is a board/committee member of AOSSM. He is a paid consultant for DePuy and Vericel. He has received payment for development of educational presentations from Liberty Surgical. F.P.T. is a board or committee member of AOSSM, AAOS, and ABOS. He owns stock options in Franklin/Keystone Biosciences and Trice Medical. Full ICMJE author disclosure forms are available for this article online, as supplementary material.

Received April 24, 2021; accepted September 17, 2021.

Address correspondence to Kevin B. Freedman, M.D., Rothman Orthopaedic Institute, Thomas Jefferson University, 825 Old Lancaster Rd., Suite 200, Bryn Mawr, PA, 19010, U.S.A. E-mail: Kevin.freedman@ rothmanortho.com

(C) 2021 THE AUTHORS. Published by Elsevier Inc. on behalf of the Arthroscopy Association of North America. This is an open access article under the CC BY-NC-ND license (http://creativecommons.org/licenses/by-nc-nd/4.0/).

2666-061X/21572

https://doi.org/10.1016/j.asmr.2021.09.011

\section{Introduction}

A s pain became the "fifth vital sign" and sustainedArelease OxyContin (Purdue Pharma, Stamford, CT) was approved for use, opioids were marketed aggressively as an effective treatment for noncancerous pain. ${ }^{1,2}$ However, excessive opioid usage is associated with increased mortality ${ }^{3,4}$ and addiction, ${ }^{5}$ which have been implicated in the current opioid epidemic. In 2020, there were nearly six times more opioid-related overdose deaths than there were in $1999 .{ }^{6}$ With patient-reported pain remaining unchanged while opioid prescription rates continued to increase, ${ }^{7}$ research has been increasingly focused on nonopioid pain management techniques.

Optimal management of postoperative pain is associated with decreased morbidity and faster recovery times, as well as improved physical function and quality of life. ${ }^{8}$ Despite efforts to minimize postoperative pain, $61 \%$ of outpatients still experience moderate/extreme pain after 
discharge. 9 Some of the most painful surgeries are orthopedic procedures, with arthroscopic surgeries, such as cruciate ligament reconstruction ${ }^{10}$ and rotator cuff repair, ${ }^{11}$ considered among the most painful outpatient orthopedic surgical procedures. Because of the pain associated with these procedures, orthopedic surgeons were the third highest prescribers of opioids based on specialty in the United States, behind only primary care physicians and internists. ${ }^{12}$ The American Society of Anesthesiologists recommends the use of multimodal pain regimens to minimize opioid use and improve pain control. ${ }^{13}$ Multimodal pain management uses combinations of opioid prescriptions, nonopioid prescription, regional and local anesthesia, and nonpharmacological therapy. An effective multimodal pain management protocol should limit both postoperative pain and opioid consumption.

Four systematic reviews have evaluated randomized controlled trials (RCTs) within knee, ${ }^{14}$ hip, ${ }^{15}$ and shoulder ${ }^{16,17}$ arthroscopy. Warrender et al. ${ }^{17}$ suggests that the interscalene nerve block is the most effective analgesic for arthroscopic shoulder surgery, while Hurley et al. ${ }^{16}$ recommend nerve block adjuncts to improve pain control. In hip arthroscopy, Kunze et al. ${ }^{15}$ similarly recommend adjunct analgesia, and they suggest that local infiltration analgesia may optimize postoperative pain and opioid consumption. In knee arthroscopy, Secrist et al. ${ }^{14}$ did not determine an optimal multimodal management protocol for anterior cruciate ligament reconstruction.

In order to optimize postoperative care, treatment plans should minimize both pain and opioid consumption after arthroscopic surgery. However, few reviews have focused on postoperative opioid consumption along with pain management in arthroscopic surgery. The purpose of the study was to provide an updated review of multimodal pain management in arthroscopic surgery by evaluating pain and opioid consumption after shoulder, hip, and knee arthroscopy.

\section{Methods}

\section{Study Selection}

This systematic review was performed according to PRISMA (Preferred Reporting Items for Systematic Reviews and Meta-Analyses) guidelines. ${ }^{18}$ A comprehensive literature search was performed to identify all RCTs regarding pain management after arthroscopic surgeries of the shoulder, hip, and knee. Articles were identified from a 10-year period ranging from January 1st, 2011 through December 31st, 2020 by using the PubMed, Ovid, and CINAHL databases. The following keywords were used: opioid, pain management, multimodal, sports medicine, shoulder, hip, knee, surgery, surgical, and arthroscopy. Screening of RCTs by title and abstract was performed by two independent researchers, R.W.P. (research fellow) and P.S. (orthopaedic surgery resident), with disagreements settled by K.B.F. (attending orthopaedic surgeon).

\section{Inclusion and Exclusion Criteria}

Only RCTs that 1) were related to arthroscopic surgery of the shoulder, hip, and knee, 2) reported both postoperative pain and volume of postoperative opioid consumption, and 3) had a dependent variable focusing on multimodal pain management, were included. Interventions provided preoperatively, intraoperatively, and postoperatively were all included as well. Multimodal pain management was considered any combination of at least two of the following: education, exercise interventions, pharmaceutical medications, regional anesthesia, rehabilitative interventions (exercise, manual therapy, physical modalities), and workplace intervention. Combinations of varying medications were also considered multimodal, as has been done in several other systematic reviews and meta-analyses. ${ }^{19-22}$ Studies that 1) were not randomized controlled trials focusing on arthroscopic surgery of the shoulder, hip, and knee, 2) did not report both postoperative pain scores and postoperative opioid consumption, and 3) did not have an intervention regarding multimodal pain management, were excluded.

\section{Assessment of Study Quality}

Included studies were evaluated for bias using the Cochrane Risk of Bias tool. ${ }^{23}$ Six categories of bias assessment were used from the Cochrane Risk of Bias tool: random sequence generation, allocation concealment, blinding of participants and personnel, blinding of outcome assessment, incomplete outcome data, and selective reporting. Bias in each category was classified as high, low, or unclear.

\section{Data Collection and Abstraction}

Surgical category (shoulder, hip, knee arthroscopy), pain management intervention, descriptions of treatment groups, details regarding treatment dosages, and demographic data (age, sex, and BMI) were collected from each included study. As the primary outcome variables of this study, postoperative pain scores and volume of postoperative opioid consumption were collected from all reported postoperative time points. Statistically significant differences in postoperative pain scores and opioid consumption were noted within each included study.

\section{Statistical Analysis}

Because of differences in study intervention, patient populations, and surgical procedure, postoperative pain scores and opioid consumption were not pooled. Summary data regarding the postoperative pain scores and opioid consumption from all time points were presented. 


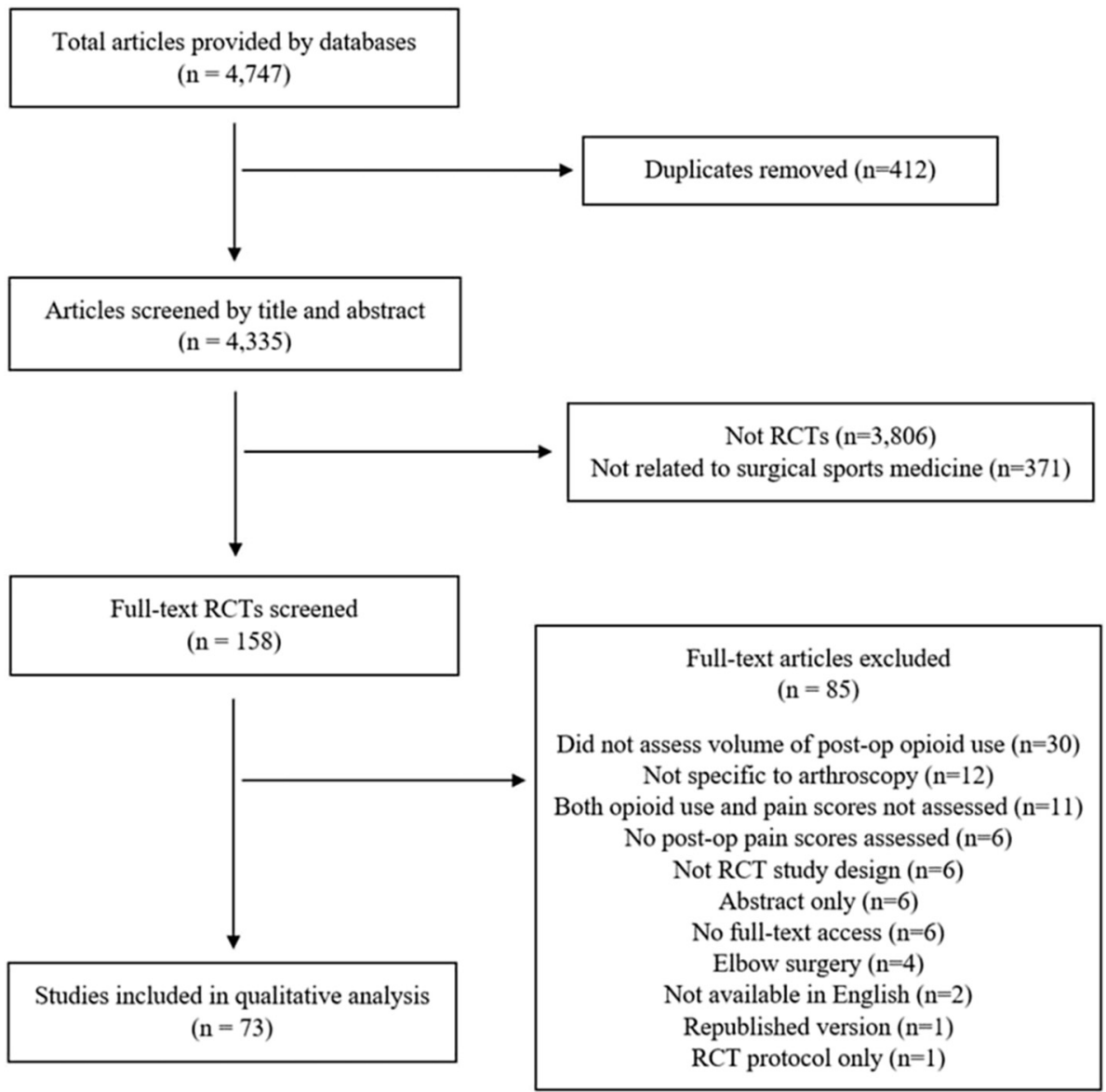

Fig 1. Flowchart of randomized controlled trial (RCT) screening process, with 73 final studies included, and the reasons for excluding 85 other articles are noted.

\section{Results}

Overall, 4,335 nonduplicate articles were screened by title and abstract for inclusion. After excluding 3,806 studies that were not RCTs and 371 that were not related to arthroscopy, 158 studies were screened by full text. Eighty-five articles were excluded based on full text, with exclusion reasons available in Fig 1 . Seventy-three RCTs assessed both postoperative pain and postoperative opioid consumption after arthroscopic procedures and were included in the final qualitative analysis.

\section{Study Quality}

The most frequent bias present in the included RCTs was incomplete outcome data, as 42 out of the 73 included RCTs ( $58 \%$ ) either did not provide data for all variables or did not provide adequate statistics, such as standard deviations and exact $P$ values (Table 1, Fig 2). Group allocation concealment was the least frequent bias, as 62 of the 73 included studies (85\%) concealed participants' group allocations, often by using sealed opaque envelopes. The rest of the average Cochrane Risk of Bias tool data is available in Fig 2, with individual studies' bias scores available in Table 1 .

\section{Shoulder Arthroscopy}

There were 37 RCTs that assessed pain and opioid consumption after shoulder arthroscopy (Table 2); 18 of the RCTs assessed nerve blocks, while 3 evaluated localized injections, 3 assessed oral medications, and 3 evaluated nonpharmacological interventions. Also, 12 RCTs isolated patients that underwent rotator cuff repair, 2 RCTs isolated patients that underwent subacromial decompression, and I RCT isolated patients that underwent Bankart repair. Finally, 20 studies showed significant differences in postoperative pain, and 21 studies found significant differences in opioid consumption.

\section{Rotator Cuff Repair}

Four studies evaluated nerve blocks for rotator cuff repair specifically. ${ }^{32,37,44,87}$ Cabaton et al. ${ }^{37}$ found that a supraclavicular nerve block with $100 \mathrm{mg}$ levobupivacaine and clonidine provided similar pain management but much less opioid consumption from 0 to 48 hours postoperatively compared to an ultrasound-guided interscalene nerve block with the same dosages. Wong et al. ${ }^{87}$ shows that an interscalene block with 20 $\mathrm{mL}$ of $.2 \%$ ropivacaine decreases opioid consumption at 
Table 1. Cochrane Risk of Bias Data for all Included Studies

\begin{tabular}{|c|c|c|c|c|c|c|c|}
\hline $\begin{array}{c}\text { Author } \\
\end{array}$ & Publication Year & $\begin{array}{c}\text { Random } \\
\text { Sequence } \\
\text { Generation } \\
\end{array}$ & $\begin{array}{c}\text { Allocation } \\
\text { Concealment }\end{array}$ & $\begin{array}{c}\text { Incomplete } \\
\text { Outcome Data }\end{array}$ & $\begin{array}{c}\text { Blind of } \\
\text { Participants } \\
\text { and Personnel }\end{array}$ & $\begin{array}{c}\text { Blinding of } \\
\text { Outcome } \\
\text { Assessment } \\
\end{array}$ & $\begin{array}{c}\text { Selective } \\
\text { Reporting }\end{array}$ \\
\hline$\overline{\text { Abdallah et al. }{ }^{24}}$ & 2016 (knee) & High & Low & High & Low & Low & Low \\
\hline Abdallah et al. ${ }^{24}$ & 2016 (shoulder) & Low & Low & Low & Low & Low & Low \\
\hline Abdallah et al. ${ }^{25}$ & 2019 & Low & Low & Low & Low & Low & Low \\
\hline Abdallah et al. ${ }^{26}$ & 2020 & Unclear & Low & High & Low & Low & Low \\
\hline Ahn et al. ${ }^{27}$ & 2016 & Low & Low & Low & Low & Low & Low \\
\hline Aksu et al. ${ }^{28}$ & 2015 & Low & Unclear & Low & High & Low & High \\
\hline Amin et al. ${ }^{29}$ & 2011 & Low & High & High & Unclear & Unclear & High \\
\hline Arti and Mehdinasab ${ }^{30}$ & 2011 & High & Low & High & Low & Low & Low \\
\hline Auyong et al. ${ }^{31}$ & 2018 & Low & Low & Low & Low & Low & Low \\
\hline Baessler et al. $^{32}$ & 2020 & Low & Low & Low & High & Unclear & Low \\
\hline Bailey et al. ${ }^{33}$ & 2019 & Low & Unclear & Low & High & Low & Low \\
\hline Behrends et al. ${ }^{34}$ & 2018 & Low & Low & High & Low & Low & High \\
\hline Bengisun et al. ${ }^{35}$ & 2014 & Low & Low & High & Low & Low & Low \\
\hline Bjørnholdt et al. ${ }^{36}$ & 2014 & Unclear & Low & High & Low & Low & Low \\
\hline Cabaton et al. ${ }^{37}$ & 2019 & Low & Low & High & High & Low & Low \\
\hline Choromanski et al. ${ }^{38}$ & 2015 & Low & Low & Low & Low & Low & Low \\
\hline Cho et al. ${ }^{39}$ & 2011 & Low & High & High & High & Unclear & High \\
\hline Cogan et al. ${ }^{40}$ & 2020 & Low & Low & High & Low & Low & High \\
\hline DeMarco et al. ${ }^{41}$ & 2011 & Low & Low & High & Low & Low & Low \\
\hline Espelund et al. ${ }^{42}$ & 2014 & Low & Low & High & Low & Low & Low \\
\hline Espelund et al. ${ }^{43}$ & 2014 & Low & Low & High & Low & Low & Low \\
\hline Faria-Silva et al. ${ }^{44}$ & 2016 & Low & Unclear & High & High & Low & Low \\
\hline Glomset et al. ${ }^{45}$ & 2020 & Low & Unclear & Low & High & Unclear & Low \\
\hline Hanson et al. ${ }^{46}$ & 2013 & Low & Low & High & Low & Low & Low \\
\hline Hartwell et al. ${ }^{47}$ & 2020 & High & High & Low & High & High & Low \\
\hline Hsu et al. ${ }^{48}$ & 2013 & Low & Low & Low & Low & Low & Low \\
\hline Jeske et al. ${ }^{49}$ & 2011 & Low & Low & High & Low & Low & High \\
\hline Kager et al. ${ }^{50}$ & 2011 & Low & Low & High & Low & Unclear & Low \\
\hline Kahlenberg et al. ${ }^{51}$ & 2017 & Unclear & Low & High & Low & Unclear & Low \\
\hline Kahn et al. ${ }^{52}$ & 2018 & Low & Low & Low & Low & Low & Low \\
\hline Kang et al. ${ }^{53}$ & 2018 & Low & Low & High & Low & Low & Low \\
\hline Kang et al. ${ }^{54}$ & 2019 & Low & Low & High & Low & Low & Low \\
\hline Kataria et al. ${ }^{55}$ & 2019 & Low & Low & High & Low & Low & Low \\
\hline Keller et al. ${ }^{56}$ & 2019 & Low & Low & Unclear & Low & Low & Unclear \\
\hline Khashan et al. ${ }^{57}$ & 2016 & Low & Low & Low & Low & Low & Low \\
\hline Kim et al. $^{58}$ & 2019 & Low & Low & Low & Low & Low & Low \\
\hline Ko et al. ${ }^{59}$ & 2013 & Low & Low & High & Low & Low & Low \\
\hline Koltka et al. ${ }^{60}$ & 2011 & Low & Unclear & High & High & Low & High \\
\hline Kraeutler et al. ${ }^{61}$ & 2015 & Unclear & High & High & High & High & Low \\
\hline Lee et al. ${ }^{62}$ & 2015 & Low & Low & High & Unclear & Unclear & Low \\
\hline Lee et al. ${ }^{62}$ & 2012 & Low & Low & High & Low & Low & High \\
\hline Lierz et al. ${ }^{63}$ & 2012 & Low & Low & High & Low & Low & Low \\
\hline Lu et al. ${ }^{64}$ & 2017 & Low & Low & Low & Low & Low & Low \\
\hline Lynch et al. ${ }^{65}$ & 2019 & Low & Low & Low & Low & Low & Low \\
\hline Mahure et al. ${ }^{66}$ & 2017 & Low & Low & Low & Low & Low & Low \\
\hline Mardani-Kivi et al. ${ }^{67}$ & 2016 & Low & Low & Low & Low & Low & Low \\
\hline Mardani-Kivi et al. ${ }^{68}$ & 2013 & Low & Low & Low & Low & Low & Low \\
\hline Marinković et al. ${ }^{69}$ & 2016 & Unclear & Unclear & High & Unclear & Unclear & High \\
\hline McHardy et al. ${ }^{70}$ & 2020 & Low & Low & Low & Low & Low & Low \\
\hline Merivirta et al. ${ }^{71}$ & 2012 & Low & Low & High & Low & Low & Low \\
\hline Merivirta et al. ${ }^{71}$ & 2013 & Low & Low & High & Low & Unclear & Low \\
\hline Mitra et al. ${ }^{72}$ & 2011 & Low & Low & Low & Low & Low & High \\
\hline Moyano et al. ${ }^{73}$ & 2016 & Low & Low & Low & Low & Low & Low \\
\hline Neuts et al. ${ }^{74}$ & 2018 & Low & Low & High & Low & Low & Low \\
\hline Oh et al. ${ }^{75}$ & 2018 & Low & Low & High & Low & Low & Low \\
\hline Premkumar et al. ${ }^{76}$ & 2016 & Low & Low & High & Low & Low & Low \\
\hline Purcell et al. ${ }^{77}$ & 2019 & High & Low & Low & High & Low & Low \\
\hline Reda et al. $^{78}$ & 2016 & Low & Low & High & High & Low & Low \\
\hline Sanel et al. ${ }^{79}$ & 2016 & Unclear & High & Low & Low & Low & Low \\
\hline Saritas et al. ${ }^{80}$ & 2015 & Low & Low & High & Low & Low & Low \\
\hline Sayin et al. ${ }^{76}$ & 2015 & Low & Low & Low & Unclear & Unclear & High \\
\hline
\end{tabular}


Table 1. Continued

\begin{tabular}{|c|c|c|c|c|c|c|c|}
\hline Author & Publication Year & $\begin{array}{c}\text { Random } \\
\text { Sequence } \\
\text { Generation }\end{array}$ & $\begin{array}{c}\text { Allocation } \\
\text { Concealment }\end{array}$ & $\begin{array}{c}\text { Incomplete } \\
\text { Outcome Data }\end{array}$ & $\begin{array}{c}\text { Blind of } \\
\text { Participants } \\
\text { and Personnel }\end{array}$ & $\begin{array}{l}\text { Blinding of } \\
\text { Outcome } \\
\text { Assessment }\end{array}$ & $\begin{array}{l}\text { Selective } \\
\text { Reporting }\end{array}$ \\
\hline Schwartzberg et al. ${ }^{81}$ & 2013 & Low & Low & High & Low & Low & Low \\
\hline Shlaifer et al. ${ }^{82}$ & 2017 & Low & Low & High & High & Low & Unclear \\
\hline Spence et al. ${ }^{83}$ & 2011 & Low & Low & Low & Low & Low & Low \\
\hline Syed et al. ${ }^{84}$ & 2018 & Low & Low & Low & Low & Low & Unclear \\
\hline Thapa et al. ${ }^{66}$ & 2016 & Unclear & Low & High & Low & Low & High \\
\hline Tompkins et al. ${ }^{85}$ & 2011 & Low & Low & High & Low & Low & High \\
\hline Westergaard et al. ${ }^{86}$ & 2014 & Unclear & Low & High & Low & Low & Low \\
\hline Wong et al. ${ }^{87}$ & 2016 & High & Low & Low & Low & Unclear & Low \\
\hline Xing et al. ${ }^{88}$ & 2015 & Low & Low & Low & Low & Low & Low \\
\hline Yun et al. ${ }^{89}$ & 2012 & Low & Low & High & High & High & High \\
\hline Zhang et al. ${ }^{90}$ & 2014 & Low & Low & High & Low & Low & High \\
\hline Zhou et al. $^{91}$ & 2017 & Low & Low & Low & Low & Low & Low \\
\hline
\end{tabular}

72 hours postoperatively compared to the same block with only $.1 \%$ ropivacaine. Faria-Silva et al. ${ }^{44}$ found that adding . $15 \mathrm{mg}$ clonidine to a brachial plexus block with $.33 \%$ ropivacaine does not affect pain or opioid consumption within the first day postop. Lastly, Baessler et al. $^{32}$ found that the addition of liposomal bupivacaine in an interscalene nerve block leads to less opioid consumption for several days after surgery. Baessler et al. ${ }^{32}$ also showed that providing dexamethasone with liposomal bupivacaine and conventional bupivacaine may decrease postoperative pain, while providing comparable opioid consumption. However, this difference in pain was only observed at postoperative day 3 , and not on days 1,2 , or 4 .

Three studies evaluated injections for rotator cuff repair. ${ }^{57,62,80}$ Khashan et al. ${ }^{57}$ found that adding $50 \mathrm{mg}$ ketamine to a preoperative intra-articular injection of morphine provided worse pain relief than morphine alone from 0-2 weeks postoperatively, while morphine also provides better pain relief than a saline control from 0 to 2 weeks. However, all three groups had comparable pain relief at 3 months, and no differences in opioid consumption. Saritas et al. ${ }^{80}$ found that an intra-articular injection with 1,000 $\mathrm{mg}$ of magnesium sulfate decreases pain from postoperative hours 1-12, while also significantly decreasing opioid consumption. Lastly, Lee et al. ${ }^{62}$ showed that local analgesic injections of bupivacaine and lidocaine used in the glenohumeral joint, in the subacromial space, and in both spaces all provide similar pain relief and opioid consumption.

\section{Knee Arthroscopy}

Twenty-eight RCTs assessed pain and opioid consumption after knee arthroscopy (Table 3). Eleven studies evaluated various types of nerve blocks, while 8 compared local injections and 2 assessed nonpharmacological intervention. Eleven RCTs isolated patients that underwent anterior cruciate ligament (ACL) reconstruction, and 5 isolated patients that underwent meniscectomy. Of these studies, 19 studies

\section{Risk of Bias for Included Randomized Controlled Trials}

Fig 2. Cochrane Risk of Bias tool categorical scores of all included randomized controlled trials (RCTs). Red denotes high risk. Blue denotes unclear. Green denotes low risk.

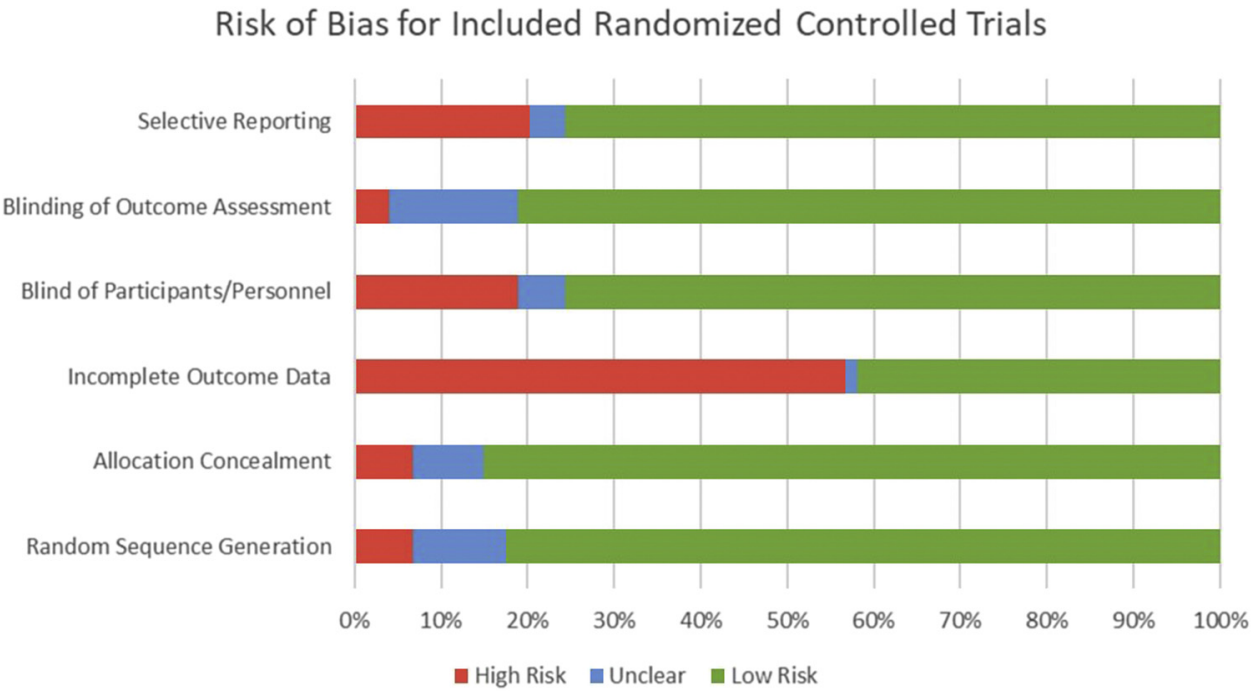


Table 2. Treatment Provided, Patient Population, Postoperative Pain, and Postoperative Opioid Consumption Summarized from all Included RCTs Regarding Shoulder Arthroscopy

\begin{tabular}{|c|c|c|c|c|c|c|c|c|c|}
\hline $\begin{array}{c}\text { Author, } \\
\text { Publication } \\
\text { Year }\end{array}$ & $\begin{array}{l}\text { Level of } \\
\text { Evidence }\end{array}$ & Surgical Procedure & Intervention & Treatment Groups & Dosage & $\begin{array}{l}\text { Patients } \\
\quad(n)\end{array}$ & $\begin{array}{c}\text { Age } \\
\text { (years) }\end{array}$ & $\begin{array}{l}\text { Post-Op Pain } \\
\text { Differences }\end{array}$ & $\begin{array}{l}\text { Post-Op Opioid } \\
\text { Consumption } \\
\text { Differences }\end{array}$ \\
\hline \multirow[t]{2}{*}{$\begin{array}{l}\text { Cabaton et al., } \\
2019^{37}\end{array}$} & \multirow[t]{2}{*}{1} & \multirow[t]{2}{*}{ Rotator cuff repair } & \multirow[t]{2}{*}{ Nerve block } & $\begin{array}{l}\text { SCB: Supraclavicular } \\
\text { nerve block }\end{array}$ & $\begin{array}{l}100 \text { mg levobupivacaine with } \\
\text { clonidine }\end{array}$ & 52 & 57 & \multirow{2}{*}{$\begin{array}{l}\text { NRS scale: } \mathrm{SCB}= \\
\text { ISB, from } 0 \text { to } 48 \\
\text { hours }\end{array}$} & \multirow{2}{*}{$\begin{array}{c}\text { Total morphine } \\
\text { consumed: SCB < ISB, } \\
\text { from } 0 \text { to } 48 \text { hours }\end{array}$} \\
\hline & & & & $\begin{array}{l}\text { ISB: Ultrasound-guided } \\
\text { interscalene nerve block }\end{array}$ & $\begin{array}{l}100 \mathrm{mg} \text { levobupivacaine with } \\
\text { clonidine }\end{array}$ & 51 & 58 & & \\
\hline \multirow[t]{2}{*}{ Wong et al., $2016^{87}$} & \multirow[t]{2}{*}{2} & \multirow[t]{2}{*}{ Rotator cuff repair } & \multirow[t]{2}{*}{ Nerve Block } & $\begin{array}{l}.1 \%: \text { Phrenic nerve } \\
\text { block, } .1 \% \text { ropivacaine }\end{array}$ & $\begin{array}{l}\text { Ultrasound-guided } \\
\text { interscalene block with } 20 \\
\text { mL of } .1 \% \text { ropivacaine }\end{array}$ & 18 & 48.3 & \multirow[t]{2}{*}{$\begin{array}{l}\text { DVPRS: } .1 \%=.2 \% \text {, } \\
\text { at } 30 \text { min and } 1 \text { hour }\end{array}$} & \multirow{4}{*}{$\begin{array}{c}\text { PACU fentanyl } \\
\text { consumption, and } \\
\text { codeine equivalents at } \\
72 \text { hours: } .1 \%=.2 \% \text {, } \\
\text { in PACU. } .1 \%>.2 \% \text {, } \\
\text { at } 72 \text { hours post-block } \\
\text { Doses of rescue } \\
\text { analgesic: } \mathrm{LA}+\mathrm{CL}=\mathrm{LA}, \\
\text { total consumption }\end{array}$} \\
\hline & & & & $\begin{array}{l}.2 \%: \text { Phrenic nerve } \\
\text { block, } .2 \% \text { ropivacaine }\end{array}$ & $\begin{array}{l}\text { Ultrasound-guided } \\
\text { interscalene block with } 20 \\
\mathrm{~mL} \text { of } .2 \% \text { ropivacaine }\end{array}$ & 19 & 40.5 & & \\
\hline \multirow[t]{2}{*}{$\begin{array}{l}\text { Faria-Silva et al., } \\
\qquad 2016^{44}\end{array}$} & \multirow[t]{2}{*}{1} & \multirow[t]{2}{*}{ Rotator cuff repair } & \multirow[t]{2}{*}{ Nerve block } & $\begin{array}{c}\text { LA + CL: Brachial plexus } \\
\text { block with ropivacaine } \\
\text { and clonidine }\end{array}$ & $\begin{array}{l}30 \mathrm{~mL} \text { of } .33 \% \text { ropivacaine } \\
\text { and } .15 \mathrm{mg} \text { clonidine }\end{array}$ & 26 & $52 \pm 11$ & \multirow[t]{2}{*}{$\begin{array}{l}\text { NRS: } \mathrm{LA}+\mathrm{CL}=\mathrm{LA}, \\
\text { from 6-24 hours }\end{array}$} & \\
\hline & & & & $\begin{array}{l}\text { LA (local anesthetics): } \\
\text { Brachial plexus block } \\
\text { with ropivacaine }\end{array}$ & $30 \mathrm{~mL}$ of $.33 \%$ ropivacaine & 24 & $54 \pm 10$ & & \\
\hline \multirow[t]{3}{*}{$\begin{array}{l}\text { Auyong et al., } \\
2018^{31}\end{array}$} & \multirow[t]{3}{*}{1} & \multirow{3}{*}{$\begin{array}{l}\text { Rotator cuff repair } \\
(90 \%) \text { or Bankart } \\
\text { repair }(10 \%)\end{array}$} & \multirow[t]{3}{*}{ Nerve block } & $\begin{array}{l}\text { ISB: Interscalene nerve } \\
\text { block }\end{array}$ & $15 \mathrm{~mL}$ of $.5 \%$ ropivacaine & 63 & $54 \pm 13$ & \multirow{3}{*}{$\begin{array}{l}\text { NRS: ISB }=S C B= \\
\text { SSB, in PACU and at } \\
1 \text { hour }\end{array}$} & \multirow{3}{*}{$\begin{array}{l}\text { Fentanyl consumption: } \\
\mathrm{ISB}=\mathrm{SCB}=\mathrm{SSB} \text {, in } \\
\mathrm{PACU} \text { and at } 1 \text { hour }\end{array}$} \\
\hline & & & & $\begin{array}{l}\text { SCB: Supraclavicular } \\
\text { nerve block }\end{array}$ & $15 \mathrm{~mL}$ of $.5 \%$ ropivacaine & 63 & $53 \pm 14$ & & \\
\hline & & & & $\begin{array}{l}\text { SSB: Suprascapular } \\
\text { nerve block }\end{array}$ & $15 \mathrm{~mL}$ of $.5 \%$ ropivacaine & 63 & $55 \pm 14$ & & \\
\hline \multirow[t]{4}{*}{ Kang $2018^{53}$} & \multirow[t]{4}{*}{2} & \multirow{4}{*}{$\begin{array}{l}85 \% \text { rotator cuff } \\
\text { repair, } 11 \% \\
\text { Bankart repair, } \\
4 \% \text { other }\end{array}$} & \multirow[t]{4}{*}{ Nerve block } & Control & $50 \mathrm{~mL}$ of $.9 \%$ normal saline & 18 & $47.8 \pm 14.4$ & \multirow{4}{*}{$\begin{array}{l}\text { VAS: DEX } 2.0< \\
\text { control only, at } 12 \\
\text { hour. All groups are } \\
\text { equal at } 6 \text { hours and } \\
24 \text { hours }\end{array}$} & \multirow{4}{*}{$\begin{array}{c}\text { Opioid Consumption: } \\
\text { *DEX } 2.0<\text { DEX 1.0, } \\
\text { DEX .5, and control, at } \\
24 \text { hour. All groups are } \\
\text { equal at } 6 \text { hour and } 12 \\
\text { hour }\end{array}$} \\
\hline & & & & $\begin{array}{c}\text { DEX .5: } \\
\text { Dexmedetomidine }\end{array}$ & $\begin{array}{l}\text { IV DEX . } 5 \mu \mathrm{g} / \mathrm{kg} \text { added to } 50 \\
\mathrm{~mL} \text { of } .9 \% \text { normal saline }\end{array}$ & 18 & $53.7 \pm 13.6$ & & \\
\hline & & & & $\begin{array}{c}\text { (DEX), } .5 \mu \mathrm{g} / \mathrm{kg} \\
\text { DEX 1.0: DEX- } 1.0 \mu \mathrm{g} / \mathrm{kg}\end{array}$ & $\begin{array}{l}\text { IV DEX } 1.0 \mu \mathrm{g} / \mathrm{kg} \text { added to } 50 \\
\mathrm{~mL} \text { of } .9 \% \text { normal saline }\end{array}$ & 18 & $49.7 \pm 12.5$ & & \\
\hline & & & & DEX 2.0: DEX- $2.0 \mu \mathrm{g} / \mathrm{kg}$ & $\begin{array}{l}\text { IV DEX } 2.0 \mu \mathrm{g} / \mathrm{kg} \text { added to } 50 \\
\mathrm{~mL} \text { of } .9 \% \text { normal saline }\end{array}$ & 18 & $52.9 \pm 10.5$ & & \\
\hline \multirow[t]{2}{*}{ Kataria $2019^{55}$} & \multirow[t]{2}{*}{1} & \multirow[t]{2}{*}{$\begin{array}{l}73 \% \text { Bankart } \\
\text { repair, } 27 \% \\
\text { rotator cuff repair }\end{array}$} & \multirow[t]{2}{*}{ Nerve block } & $\begin{array}{l}\text { A: Ultrasound-guided } \\
\text { interscalene block (ISB) } \\
\text { with dexmedetomidine } \\
\text { (DXM) }\end{array}$ & $\begin{array}{c}20 \mathrm{~mL} .5 \% \text { ropivacaine }+2 \\
\mathrm{~mL} \text { saline containing DXM } .5 \\
\mathrm{mcg} / \mathrm{kg}\end{array}$ & 30 & $30.1 \pm 10.9$ & \multirow{2}{*}{$\begin{array}{c}\text { VAS: DXA < DXM, } \\
\text { at } 24 \text { hours DXM }= \\
\text { DXA at } 30 \text { minutes, } \\
1 \text { hour, } 2 \text { hours, } 3 \\
\text { hours, } 6 \text { hours, and } \\
12 \text { hours }\end{array}$} & $\begin{array}{c}\text { Analgesic } \\
\text { consumption: DXA } \\
\text { < DXM, total } \\
\text { consumption }\end{array}$ \\
\hline & & & & $\begin{array}{l}\text { A: Ultrasound-guided } \\
\text { interscalene block (ISB) } \\
\text { with dexamethasone } \\
\text { (DXA) }\end{array}$ & $\begin{array}{c}20 \mathrm{~mL} .5 \% \text { ropivacaine }+2 \\
\mathrm{~mL} \text { saline containing DXA } .5 \\
\mathrm{mcg} / \mathrm{kg}\end{array}$ & 30 & $30.2 \pm 11.7$ & & \\
\hline Neuts $2018^{74}$ & 1 & $\begin{array}{l}\text { Rotator cuff } \\
\text { repair }+ \\
\text { decompression }\end{array}$ & Nerve block & $\begin{array}{l}\text { Interscalene brachial } \\
\text { plexus nerve block } \\
\text { (ISBPNB) }\end{array}$ & $20 \mathrm{~mL} .75 \%$ ropivacaine & 50 & $54 \pm 10$ & $\begin{array}{l}\text { NRS: ISBPNB < } \\
\text { SSB } / A X \text {, from 0-8 } \\
\text { hours ISBPNB }=\end{array}$ & $\begin{array}{c}\text { Oxycodone } \\
\text { Equivalents: ISBPNB } \\
<\text { SSB/AX, from } 0-8\end{array}$ \\
\hline & & $\begin{array}{c}(38 \%) \text { and } \\
\text { subacromial } \\
\text { decompression } \\
(31 \%), \text { other } \\
(31 \%)\end{array}$ & & $\begin{array}{l}\text { Suprascapular }+ \text { axillary } \\
\text { nerve bock (SSB/AX) }\end{array}$ & $10 \mathrm{~mL} .75 \%$ ropivacaine & 48 & $51 \pm 10$ & $\begin{array}{l}\text { SSB/AX from } 8-24 \\
\text { hours }\end{array}$ & $\begin{array}{l}\text { hours ISBPNB }=\mathrm{SBB} / \\
\mathrm{AX} \text { from } 8-24 \text { hours }\end{array}$ \\
\hline $\operatorname{Kim} 2019^{58}$ & 1 & $\begin{array}{l}\text { Rotator cuff repair } \\
(48 \%) \text {, other }\end{array}$ & Nerve Block & $\begin{array}{l}\text { Superior trunk block } \\
\text { (STB) }\end{array}$ & $15 \mathrm{~mL} .5 \%$ bupivicaine & 62 & 51.5 & $\begin{array}{l}\text { NRS: STB = ISBPNB, } \\
\text { from 1-48 hours }\end{array}$ & $\begin{array}{l}\text { Morphine Equivalents: } \\
\text { STB = ISBPNB, from }\end{array}$ \\
\hline & & $(52 \%)$ & & $\begin{array}{l}\text { Interscalene brachial } \\
\text { plexus nerve block } \\
\text { (ISBPNB) }\end{array}$ & $15 \mathrm{~mL} .5 \%$ bupivicaine & 63 & 50 & & 0-48 hours \\
\hline
\end{tabular}




\begin{tabular}{|c|c|c|c|c|c|c|c|c|c|}
\hline $\begin{array}{l}\text { Author, } \\
\text { Publication } \\
\text { Year }\end{array}$ & $\begin{array}{l}\text { Level of } \\
\text { Evidence }\end{array}$ & Surgical Procedure & Intervention & Treatment Groups & Dosage & $\begin{array}{l}\text { Patients } \\
\quad(n)\end{array}$ & $\begin{array}{c}\text { Age } \\
\text { (years) }\end{array}$ & $\begin{array}{l}\text { Post-Op Pain } \\
\text { Differences }\end{array}$ & $\begin{array}{l}\text { Post-Op Opioid } \\
\text { Consumption } \\
\text { Differences }\end{array}$ \\
\hline Choromanski $2015^{38}$ & 2 & $\begin{array}{c}\text { Bankart repair } \\
(17 \%), \text { superior } \\
\text { labrum anterior } \\
\text { and posterior } \\
\text { repair }(30 \%), \\
\text { arthroscopic } \\
\text { rotator cuff repair } \\
(30 \%), \text { other } \\
(23 \%)\end{array}$ & $\begin{array}{c}\text { Continuous Nerve } \\
\text { Block }\end{array}$ & $\begin{array}{c}\text { Continuous interscalene } \\
\text { nerve block catheter } \\
\text { with .125\% bupivacaine } \\
\text { Continuous interscalene } \\
\text { brachial plexus nerve } \\
\text { block catheter with } .2 \% \\
\text { ropivacaine }\end{array}$ & $\begin{array}{c}400 \mathrm{~mL} .125 \% \text { bupivacaine } \\
@ 6 \mathrm{~mL} / \mathrm{h} \\
400 \mathrm{~mL} .2 \% \text { ropivacaine @ } 6 \\
\mathrm{~mL} / \mathrm{h}\end{array}$ & 14 & $\begin{array}{l}54 \pm 18.8 \\
48.2 \pm 17.7\end{array}$ & $\begin{array}{l}\text { VAS: Ropivacaine }= \\
\text { bupivacaine, on } \\
\text { postop day } 1\end{array}$ & $\begin{array}{c}\text { Oxycodone } \\
\text { Equivalents: } \\
\text { Ropivacaine }= \\
\text { Bupivacaine, from } 0 \text { to } \\
24 \text { hours }\end{array}$ \\
\hline $\begin{array}{l}\text { Abdallah et al., } \\
2020^{26}\end{array}$ & 1 & $\begin{array}{c}\text { Acromioplasty } \\
(29 \%), \text { rotator cuff } \\
\text { repair }(22 \%), \\
\text { biceps tenodesis } \\
(11 \%), \text { other } \\
(37 \%)\end{array}$ & Nerve block & $\begin{array}{l}\text { Interscalene block (ISB) } \\
\text { Subomohyoid anterior } \\
\text { suprascapular block } \\
\text { (SASB) }\end{array}$ & $\begin{array}{l}15 \mathrm{~mL} .5 \% \text { ropivacaine with } \\
\text { epinephrine } 1: 200,000 \\
15 \mathrm{~mL} .5 \% \text { ropivacaine with } \\
\text { epinephrine } 1: 200,000\end{array}$ & $\begin{array}{l}69 \\
67\end{array}$ & $\begin{array}{l}40 \pm 15 \\
46 \pm 15\end{array}$ & $\begin{array}{l}\text { VAS: ISB = SASB, } \\
\text { from } 0 \text { to } 24 \text { hours }\end{array}$ & $\begin{array}{c}\text { Morphine Equivalent } \\
\text { Consumption: ISB < } \\
\text { SASB, in PACU. ISB = } \\
\text { SASB, until } 24 \text { hours }\end{array}$ \\
\hline $\begin{array}{l}\text { Baessler et al., } \\
2020^{32}\end{array}$ & 1 & $\begin{array}{l}\text { Rotator cuff repair, } \\
\text { with frequent } \\
\text { concomitant } \\
\text { biceps tenodesis } \\
(46 \%) \text { and biceps } \\
\text { tenotomy }(46 \%)\end{array}$ & Nerve block & $\begin{array}{c}\text { LBD group: Liposomal } \\
\text { bupivacaine (LB) }+ \\
\text { dexamethasone }+ \\
\text { conventional } \\
\text { bupivacaine } \\
\text { LB group: Liposomal } \\
\text { bupivacaine }+ \\
\text { conventional } \\
\text { bupivacaine } \\
\text { Control group: } \\
\text { Conventional } \\
\text { bupivacaine }+ \\
\text { dexamethasone }\end{array}$ & $\begin{array}{l}15 \mathrm{~mL} .5 \% \text { bupivacaine, } 10 \\
\mathrm{~mL}(133 \mathrm{mg}) \mathrm{LB}, .4 \mathrm{~mL}(4 \\
\mathrm{mg}) \text { dexamethasone, and } 5 \\
\mathrm{~mL} \text { saline solution } \\
\\
15 \mathrm{~mL} .5 \% \text { bupivacaine, } 10 \\
\mathrm{~mL}(133 \mathrm{mg}) \mathrm{LB} \text {, and } 5.4 \mathrm{~mL} \\
\text { saline } \\
\text { } \\
30 \mathrm{~mL} .5 \% \text { bupivacaine and } \\
.4 \mathrm{~mL}(4 \mathrm{mg}) \text { of preservative- } \\
\text { free dexamethasone }\end{array}$ & 26 & $56.9 \pm 9.6$ & $\begin{array}{l}\text { VAS: } \mathrm{LBD}<\mathrm{LB} \text {, day } \\
\text { 3. All groups were } \\
\text { similar at all other } \\
\text { time points, days } 1-4\end{array}$ & $\begin{array}{c}\text { Oral Morphine } \\
\text { Milligram Equivalents: } \\
\text { LB }=\text { LBD, days } 1-4 \text {. } \\
\text { *LB }<\text { Control, day } 2 . \\
\text { LB }<\text { Control, day } 3 . \\
\text { LB }<\text { Control, day } 2 \text {. } \\
\text { *LB }<\text { Control, day } 3\end{array}$ \\
\hline DeMarco $2011^{41}$ & 1 & $\begin{array}{l}83 \% \text { bursectomy, } \\
79 \% \text { subacromial } \\
\text { decompression, } \\
32 \% \text { rotator cuff } \\
\text { repair, and other } \\
\text { concomitant } \\
\text { procedures }\end{array}$ & Nerve block & $\begin{array}{l}\text { ISB: Preoperative } \\
\text { interscalene nerve block } \\
\text { Placebo }\end{array}$ & $\begin{array}{c}30 \mathrm{~mL} \text { of } .5 \% \text { ropivacaine } \\
100 \mathrm{~mL} \text { saline solution }\end{array}$ & 28 & & $\begin{array}{c}\text { VAS: *ISB }< \\
\text { Placebo, at } 6 \text { hr. } \\
\text { ISB = Placebo, from } \\
12-80 \text { hours }\end{array}$ & $\begin{array}{c}\text { Narcotic Pills Used: } \\
\text { ISB = Placebo, from 6- } \\
80 \text { hours }\end{array}$ \\
\hline Ko $2013^{59}$ & 2 & Acromioplasty & Nerve block & $\begin{array}{l}\text { UG SSB: Ultrasound- } \\
\text { guided suprascapular } \\
\text { nerve block } \\
\text { EG SSB: } \\
\text { Electrophysiology- } \\
\text { guided suprascapular } \\
\text { nerve block } \\
\text { Blind SSB: Suprascapular } \\
\text { nerve block using } \\
\text { anatomic landmarks }\end{array}$ & $10 \mathrm{~mL}$ of $.375 \%$ ropivacaine & 15 & $42.8 \pm 14.3$ & $\begin{array}{l}\text { VAS: UG SSB }<\text { EG } \\
\text { SSB + Blind SSB, at } \\
4 \text { hours. UG SSB }= \\
\text { EG SSB = Blind SSB, } \\
\text { from } 24-72 \text { hours }\end{array}$ & $\begin{array}{c}\text { Morphine } \\
\text { Consumption: EG } \\
\text { SSB + UG SSB }<\text { Blind } \\
\text { SSB, from } 0 \text { to } 72 \\
\text { hours }\end{array}$ \\
\hline
\end{tabular}




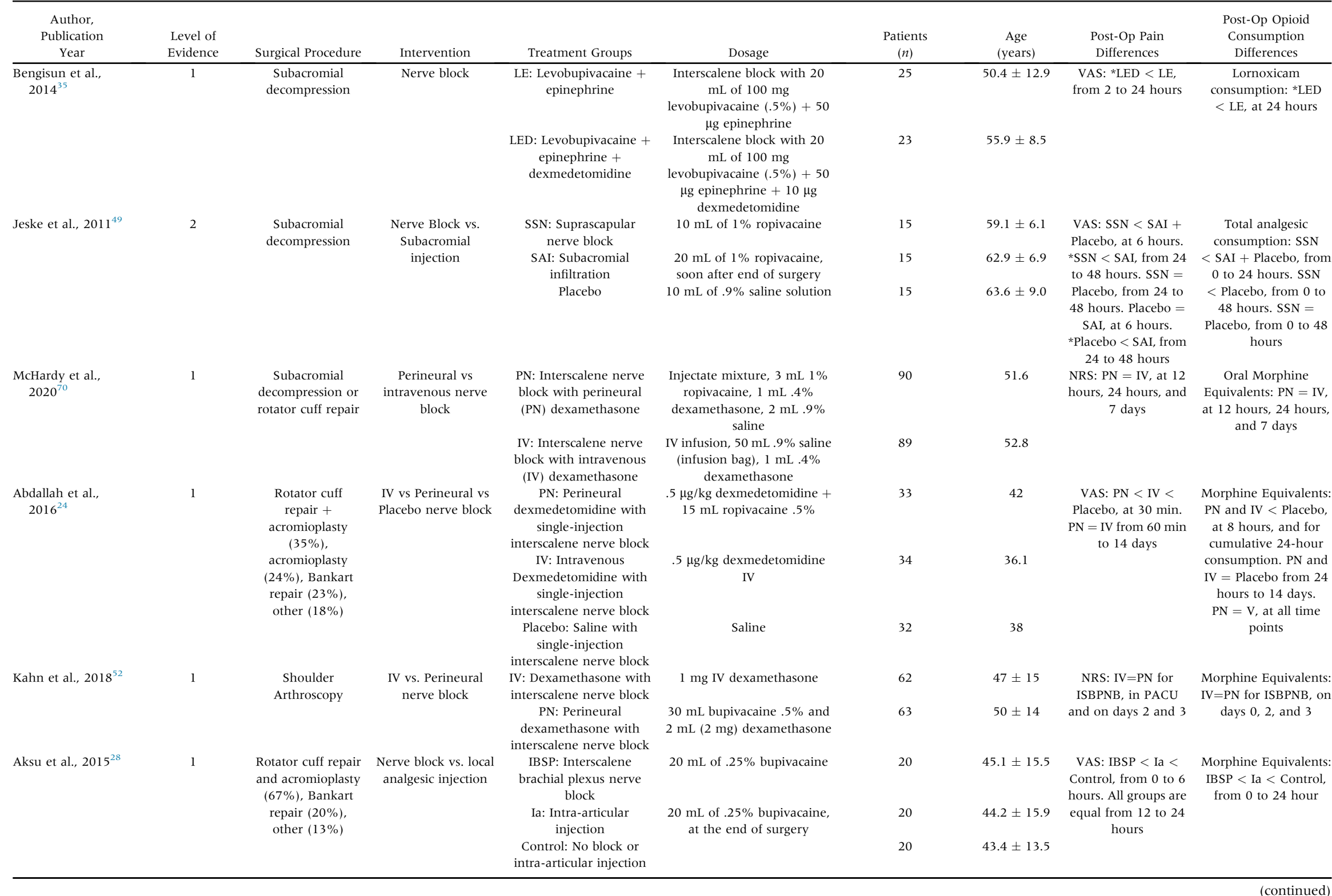




\begin{tabular}{|c|c|c|c|c|c|c|c|c|c|}
\hline $\begin{array}{l}\text { Author, } \\
\text { Publication } \\
\text { Year }\end{array}$ & $\begin{array}{l}\text { Level of } \\
\text { Evidence }\end{array}$ & Surgical Procedure & Intervention & Treatment Groups & Dosage & $\begin{array}{l}\text { Patients } \\
\qquad(n)\end{array}$ & $\begin{array}{c}\text { Age } \\
\text { (years) }\end{array}$ & $\begin{array}{l}\text { Post-Op Pain } \\
\text { Differences }\end{array}$ & $\begin{array}{l}\text { Post-Op Opioid } \\
\text { Consumption } \\
\text { Differences }\end{array}$ \\
\hline \multirow[t]{2}{*}{$\begin{array}{l}\text { Merivirta et al., } \\
2013^{71}\end{array}$} & \multirow[t]{2}{*}{1} & \multirow{2}{*}{$\begin{array}{l}54 \% \\
\text { acromioplasty, } \\
46 \% \text { rotator cuff } \\
\text { repair }\end{array}$} & \multirow[t]{2}{*}{$\begin{array}{l}\text { Subacromial } \\
\text { Catheter }\end{array}$} & Bupivacaine & $\begin{array}{l}\text { Continuous infusion of } 5 \mathrm{mg} / \\
\mathrm{mL} \text { bupivacaine, at } 2 \mathrm{~mL} / \mathrm{hr}\end{array}$ & 39 & $53 \pm 9$ & \multirow{5}{*}{$\begin{array}{l}\text { NRS: Bupivacaine }= \\
\text { Saline, from } 0 \text { to } 12 \\
\text { hours. Bupivacaine } \\
\text { < Saline, at } 18 \\
\text { hours. } \\
\text { Bupivacaine = } \\
\text { Saline, on days } 1 \text { and } \\
3 \\
\text { VAS: No catheter }< \\
\text { Catheter with saline } \\
\text { solution, } \\
\text { immediately after } \\
\text { surgery. No } \\
\text { catheter = Catheter } \\
\text { with bupivacaine, } \\
\text { and Catheter with } \\
\text { bupivacaine }=\text { No } \\
\text { catheter, } \\
\text { immediately after } \\
\text { surgery. All groups } \\
\text { are equal, from } 1 \text { to } \\
12 \text { hour }\end{array}$} & \multirow{5}{*}{$\begin{array}{c}\text { Opioid and codeine } \\
\text { consumption: } \\
\text { Bupivacaine }<\text { Saline, } \\
\text { on days } 0 \text { and } 1 . \\
\text { *Bupivacaine }<\text { Saline, } \\
\text { on day } 2 \text {. } \\
\text { Bupivacaine }=\text { Saline, } \\
\text { on day } 3 \\
\text { Oxycodone } \\
\text { consumption: All } \\
\text { groups are equal, days } \\
0-4\end{array}$} \\
\hline & & & & Saline & $\begin{array}{l}\text { Continuous infusion of } 9 \mathrm{mg} / \\
\mathrm{mL} \text { saline, at } 2 \mathrm{~mL} / \mathrm{hr}\end{array}$ & 43 & $55 \pm 6$ & & \\
\hline \multirow[t]{3}{*}{$\begin{array}{l}\text { Schwartzberg and } \\
\text { Reuss } 2013^{81}\end{array}$} & \multirow[t]{3}{*}{1} & \multirow[t]{3}{*}{ Rotator cuff repair } & \multirow[t]{3}{*}{$\begin{array}{l}\text { Subacromial } \\
\text { Catheter }\end{array}$} & $\begin{array}{l}\text { Catheter with } \\
\text { bupivacaine }\end{array}$ & $\begin{array}{l}\text { Postoperative infusion } \\
\text { catheter with } 200 \mathrm{~mL} \text { of } .5 \% \\
\text { bupivacaine without } \\
\text { epinephrine }\end{array}$ & 32 & 56 & & \\
\hline & & & & $\begin{array}{l}\text { Catheter with saline } \\
\text { solution }\end{array}$ & $\begin{array}{l}\text { Postoperative infusion } \\
\text { catheter with } 200 \mathrm{~mL} \text { of } \\
\text { sterile saline solution }\end{array}$ & 29 & 56 & & \\
\hline & & & & No catheter & & 27 & 58 & & \\
\hline \multirow[t]{3}{*}{ Kang et al., $2019^{54}$} & \multirow[t]{3}{*}{1} & \multirow{3}{*}{$\begin{array}{l}73 \% \text { rotator cuff } \\
\text { repair, } 20 \% \\
\text { Bankart repair, } \\
8 \% \text { other }\end{array}$} & \multirow[t]{3}{*}{ IV Injection } & Control: saline & $\begin{array}{l}\text { Intravenous .9\% saline } \\
\text { injection with interscalene }\end{array}$ & 22 & $46.3 \pm 16.6$ & \multirow{3}{*}{$\begin{array}{l}\text { VAS: Control }= \\
\text { D1 }=\text { D2, at } 6 \text { hours. } \\
\text { D1 }+ \text { D } 2<\text { Control, } \\
\text { at } 12 \text { hours. D2 }< \\
\text { Control }+ \text { D } 1, \text { from } \\
18 \text { to } 24 \text { hours }\end{array}$} & \multirow{3}{*}{$\begin{array}{l}\text { Morphine Equivalents: } \\
\text { D1 and D2 < Control, } \\
\text { from } 12-24 \text { hours. D2 } \\
<\text { D1 from } 18 \text { to } 24 \\
\text { hours. Control = D1 } \\
\text { and D2, at } 6 \text { hours }\end{array}$} \\
\hline & & & & D1: Dexamethasone & $\begin{array}{l}\text { nerve block, prior to surgery } \\
\text { Intravenous dexamethasone } \\
.11 \mathrm{mg} / \mathrm{kg} \text { with interscalene } \\
\text { nerve block, prior to surgery }\end{array}$ & 22 & $46.1 \pm 17.0$ & & \\
\hline & & & & $\begin{array}{l}\text { D2: Dexamethasone + } \\
\text { dexmedetomidine }\end{array}$ & $\begin{array}{c}\text { Coadministered intravenous } \\
\text { dexamethasone .11 } \mathrm{mg} / \mathrm{kg}+ \\
\text { intravenous } \\
\text { dexmedetomidine, with } \\
\text { interscalene nerve block, } \\
\text { prior to surgery }\end{array}$ & 22 & $47.4 \pm 13.5$ & & \\
\hline \multirow[t]{3}{*}{$\begin{array}{l}\text { Bjørnholdt et al., } \\
2014^{36}\end{array}$} & \multirow[t]{3}{*}{2} & \multirow{3}{*}{$\begin{array}{l}\text { Subacromial } \\
\text { decompression } \\
\text { and/or } \\
\text { acromioclavicular } \\
\text { joint resection }\end{array}$} & \multirow[t]{3}{*}{$\begin{array}{l}\text { Intravenous } \\
\text { medication }\end{array}$} & $\begin{array}{c}\text { D40: } 40 \mathrm{mg} \\
\text { Dexamethasone }\end{array}$ & $\begin{array}{l}40 \mathrm{mg} \text { dexamethasone } \\
\text { intravenously, preoperatively }\end{array}$ & 25 & $53 \pm 10$ & \multirow{3}{*}{$\begin{array}{l}\text { NRS: } \mathrm{D} 40=\mathrm{D} 8= \\
\text { Placebo, from } \\
\text { surgery until day } 3\end{array}$} & \multirow{3}{*}{$\begin{array}{c}\text { Total analgesic } \\
\text { consumption: } \\
\text { D40= D8 = Placebo, } \\
\text { from surgery until } \\
\text { day } 3\end{array}$} \\
\hline & & & & $\begin{array}{c}\text { D8: } 8 \mathrm{mg} \\
\text { dexamethasone }\end{array}$ & $\begin{array}{l}8 \mathrm{mg} \text { dexamethasone } \\
\text { intravenously, preoperatively }\end{array}$ & 26 & $55 \pm 11$ & & \\
\hline & & & & Placebo & $\begin{array}{l}\text { Placebo infused, } \\
\text { preoperatively }\end{array}$ & 22 & $49 \pm 11$ & & \\
\hline \multirow[t]{2}{*}{ Oh et al., $2018^{75}$} & \multirow[t]{2}{*}{1} & \multirow[t]{2}{*}{$\begin{array}{l}\text { Shoulder } \\
\text { Arthroscopy }\end{array}$} & \multirow[t]{2}{*}{$\begin{array}{l}\text { Intravenous Patient- } \\
\text { Controlled Analgesia } \\
\text { (PCA) }\end{array}$} & Nefopam & $\begin{array}{l}\text { PCA provided once awake, of } \\
120 \mathrm{mg} \text { nefopam, } 20 \mu \mathrm{g} / \mathrm{kg} \\
\text { fentanyl, and } 16 \mathrm{mg} \\
\text { ondansetron }\end{array}$ & 46 & $53.3 \pm 12.8$ & \multirow[t]{2}{*}{$\begin{array}{l}\text { VAS and NRS: } \\
\text { Nepofam }= \\
\text { Ketorolac, from } 10 \\
\text { min to } 48 \text { hours }\end{array}$} & \multirow[t]{2}{*}{$\begin{array}{c}\text { Total PCA: } \\
\text { Nepofam = Ketorolac, } \\
\text { from } 10 \text { min to } 24 \\
\text { hours }\end{array}$} \\
\hline & & & & Ketorolac & $\begin{array}{l}\text { PCA provided once awake, of } \\
2 \mathrm{mg} / \mathrm{kg} \text { ketorolac, } 20 \mu \mathrm{g} / \mathrm{kg} \\
\text { fentanyl, and } 16 \mathrm{mg} \\
\text { ondansetron }\end{array}$ & 46 & $51.9 \pm 11.5$ & & \\
\hline
\end{tabular}




\begin{tabular}{|c|c|c|c|c|c|c|c|c|c|}
\hline $\begin{array}{l}\text { Author, } \\
\text { Publication } \\
\text { Year }\end{array}$ & $\begin{array}{l}\text { Level of } \\
\text { Evidence }\end{array}$ & Surgical Procedure & Intervention & Treatment Groups & Dosage & $\begin{array}{l}\text { Patients } \\
\quad(n)\end{array}$ & $\begin{array}{c}\text { Age } \\
\text { (years) }\end{array}$ & $\begin{array}{l}\text { Post-Op Pain } \\
\text { Differences }\end{array}$ & $\begin{array}{l}\text { Post-Op Opioid } \\
\text { Consumption } \\
\text { Differences }\end{array}$ \\
\hline Yun et al., $2012^{89}$ & 1 & $\begin{array}{c}\text { Rotator cuff repair, } \\
\text { with SLAP lesion } \\
\text { in } 62 \% \text { and biceps } \\
\text { tear in } 17 \%\end{array}$ & $\begin{array}{c}\text { IV PCA vs. } \\
\text { Subacromial PCA }\end{array}$ & $\begin{array}{l}\text { SA-PCA: Subacromial } \\
\text { patient-controlled } \\
\text { analgesia } \\
\text { IV-PCA: Intravenous } \\
\text { patient-controlled } \\
\text { analgesia }\end{array}$ & $\begin{array}{c}150 \mathrm{~mL} \text { of } .5 \% \text { ropivacaine, } \\
\text { infused at } 2 \mathrm{~mL} / \text { hour, for } \\
\text { hours } 0-48 \text { postoperatively } \\
\text { Fentanyl }(.3-5 \mu \mathrm{g} / \mathrm{kg} / \mathrm{mL}) \\
\text { keterolac }(.03-.05 \mathrm{mg} / \mathrm{kg} / \\
\mathrm{mL}) \text {, and ondansetron }(.08 \\
\mathrm{mg} / \mathrm{mL}) \text {, infused at } 1 \mathrm{~mL} / \mathrm{hr}\end{array}$ & 30 & $54.1 \pm 11.6$ & $\begin{array}{l}\text { VAS: *SA-PCA }<\text { IV- } \\
\text { PCA, at } 1 \text { hour. SA- } \\
\text { PCA = IV-PCA, from } \\
4 \text { to } 48 \text { hours }\end{array}$ & $\begin{array}{c}\text { Rescue boluses } \\
\text { received: } \text { SA-PCA }= \\
\text { IV-PCA, from } 1 \text { to } 48 \\
\text { hours }\end{array}$ \\
\hline \multirow[t]{2}{*}{$\begin{array}{l}\text { Merivirta et al., } \\
2013^{71}\end{array}$} & \multirow[t]{2}{*}{1} & \multirow[t]{2}{*}{$\begin{array}{l}50 \% \\
\text { acriomioplasty, } \\
50 \% \text { rotator cuff } \\
\text { repair }\end{array}$} & \multirow[t]{2}{*}{ Patch vs infusion } & Fentanyl & $\begin{array}{l}12 \mu \mathrm{g} / \text { hour fentanyl patch for } \\
72 \text { hours, with } 4 \mathrm{~mL} / \mathrm{hr} \\
\text { saline infusion in a } \\
\text { subacromial manner, for } 72 \\
\text { hours }\end{array}$ & 30 & $52 \pm 9$ & \multirow[t]{2}{*}{$\begin{array}{l}\text { NRS: Fentanyl = } \\
\text { Bupivacaine, from } \\
\text { immediately after } \\
\text { surgery to day } 90\end{array}$} & \multirow[t]{2}{*}{$\begin{array}{l}\text { Rescue Analgesics } \\
\text { Used: Fentanyl = } \\
\text { Bupivacaine, from } \\
\text { recovery room to day } 3\end{array}$} \\
\hline & & & & Bupivacaine & $\begin{array}{l}2.5 \mathrm{mg} / \mathrm{mL} \text { bupivacaine } \\
\text { infusion in a subacromial } \\
\text { manner, with placebo patch, } \\
\text { for } 72 \text { hours }\end{array}$ & 30 & $54 \pm 9$ & & \\
\hline \multirow[t]{3}{*}{$\begin{array}{l}\text { Khashan et al., } \\
2016^{57}\end{array}$} & \multirow[t]{3}{*}{2} & \multirow[t]{3}{*}{ Rotator cuff repair } & \multirow[t]{3}{*}{$\begin{array}{l}\text { Intra-articular } \\
\text { injection }\end{array}$} & M: Morphine & $\begin{array}{l}20 \mathrm{mg} / 10 \mathrm{~mL} \text { morphine, } 20 \\
\text { minutes before surgery }\end{array}$ & 15 & $50.7 \pm 2.4$ & \multirow{3}{*}{$\begin{array}{c}\text { NRS: } \mathrm{M}<\mathrm{KM}+\mathrm{S} \text {, } \\
\text { on ward. } \mathrm{M}+\mathrm{S}< \\
\mathrm{KM} \text {, in PACU. } \mathrm{M}< \\
\mathrm{KM}<\mathrm{S} \text {. from } 1 \text { to } 2 \\
\text { weeks. All groups } \\
\text { are equal at } 3 \\
\text { months }\end{array}$} & \multirow{3}{*}{$\begin{array}{l}\text { Morphine Equivalents } \\
\text { until Discharge, } \\
\text { Number of } \\
\text { Paracetamol and } \\
\text { Oxycodone Capsules } \\
\text { Consumed for Weeks } \\
1 \text { and } 2: \mathrm{M}=\mathrm{KM}=\mathrm{S}, \\
\text { from } 0 \text { to } 2 \text { weeks }\end{array}$} \\
\hline & & & & $\begin{array}{l}\text { KM: Ketamine + } \\
\text { morphine }\end{array}$ & $\begin{array}{c}50 \mathrm{mg} \text { ketamine }+10 \mathrm{mg} / 10 \\
\mathrm{~mL} \text { morphine, } 20 \text { minutes } \\
\text { before surgery }\end{array}$ & 15 & $57.7 \pm 2.4$ & & \\
\hline & & & & S: Saline & $\begin{array}{l}.9 \% 10 \mathrm{~mL} \text { saline, } 20 \\
\text { minutes before surgery }\end{array}$ & 15 & $54.1 \pm 2.6$ & & \\
\hline \multirow[t]{2}{*}{ Saritas et al., $2015^{80}$} & \multirow[t]{2}{*}{1} & \multirow[t]{2}{*}{ Rotator cuff repair } & \multirow[t]{2}{*}{$\begin{array}{l}\text { Intra-articular } \\
\text { injection }\end{array}$} & Magnesium & $\begin{array}{l}1,000 \mathrm{mg} \text { magnesium sulfate } \\
(100 \mathrm{mg} / \mathrm{mL}) \text { intra- } \\
\text { articularly in } 10 \mathrm{~mL} \text { saline, at } \\
\text { end of surgery }\end{array}$ & 30 & $39.8 \pm 9.2$ & \multirow{2}{*}{$\begin{array}{l}\text { VAS: Magnesium < } \\
\text { Control, from } 1 \text { to } 12 \\
\text { hours. Magnesium }= \\
\text { Control, from } 18 \text { to } \\
24 \text { hours }\end{array}$} & \multirow[t]{2}{*}{$\begin{array}{c}\text { Total PCA Morphine: } \\
{ }^{*} \text { Magnesium < } \\
\text { Control, total } \\
\text { consumption }\end{array}$} \\
\hline & & & & Control & $\begin{array}{l}10 \mathrm{~mL} \text { IV saline, at end of } \\
\text { surgery }\end{array}$ & 30 & $41.6 \pm 10.4$ & & \\
\hline \multirow[t]{3}{*}{ Lee et al., $2015^{62}$} & \multirow[t]{3}{*}{1} & \multirow[t]{3}{*}{ Rotator cuff repair } & \multirow[t]{3}{*}{$\begin{array}{l}\text { Local analgesic } \\
\text { injection }\end{array}$} & $\begin{array}{c}\text { GJ: Glenohumeral joint } \\
\text { injection }\end{array}$ & $\begin{array}{l}20 \mathrm{~mL} \text { bupivacaine }+10 \mathrm{~mL} \\
\text { lidocaine, postoperatively }\end{array}$ & 40 & 57.2 & \multirow{3}{*}{$\begin{array}{l}\text { VAS: } \mathrm{GJ}=\mathrm{SS}= \\
\mathrm{GJ}+\mathrm{SS}, \text { from } 20 \\
\text { min to } 24 \text { hours }\end{array}$} & \multirow{3}{*}{$\begin{array}{c}\text { Boluses of Rescue } \\
\text { Analgesic: } \mathrm{GJ}=\mathrm{SS}= \\
\text { GJ }+ \text { SS, from } 1 \text { to } 24 \\
\text { hours }\end{array}$} \\
\hline & & & & $\begin{array}{c}\text { SS: Subacromial space } \\
\text { injection }\end{array}$ & $\begin{array}{l}20 \mathrm{~mL} \text { bupivacaine }+10 \mathrm{~mL} \\
\text { lidocaine, postoperatively }\end{array}$ & 42 & 58.1 & & \\
\hline & & & & $\begin{array}{l}\text { GJ+SS: Glenohumeral } \\
\text { joint + subacromial } \\
\text { space injection }\end{array}$ & $\begin{array}{c}10 \mathrm{~mL} \text { bupivacaine }+5 \mathrm{~mL} \\
\text { lidocaine in each of the two } \\
\text { injection sites, } \\
\text { postoperatively }\end{array}$ & 39 & 58.6 & & \\
\hline \multirow[t]{2}{*}{ Lu et al., $2017^{64}$} & \multirow[t]{2}{*}{1} & \multirow[t]{2}{*}{$\begin{array}{l}\text { Shoulder } \\
\text { arthroscopy }\end{array}$} & \multirow[t]{2}{*}{ Infusion } & $\begin{array}{l}\text { SD: Sufentanil }+ \\
\text { dexmedetomidine }\end{array}$ & $\begin{array}{c}.04 \mu \mathrm{g} / \mathrm{kg} / \mathrm{h} \text { Sufenanil }+.06 \\
\mu \mathrm{g} / \mathrm{kg} / \mathrm{h} \text { Dexmedetomidine, } \\
\text { postoperatively }\end{array}$ & 75 & $65.5 \pm 5.3$ & \multirow[t]{2}{*}{$\begin{array}{l}\text { VAS: } \mathrm{SD}<\mathrm{S} \text { from } 6 \\
\text { to } 48 \text { hours }\end{array}$} & $\begin{array}{c}\text { Amount of Rescue } \\
\text { Analgesia, and } \\
\text { Analgesic Liquid Pump }\end{array}$ \\
\hline & & & & S: Sufentanil only & $\begin{array}{l}.04 \mu \mathrm{g} / \mathrm{kg} / \mathrm{h} \text { Sufenanil, } \\
\text { postoperatively }\end{array}$ & 76 & $65 \pm 5.8$ & & $\begin{array}{l}\text { Volume: } \mathrm{SD}<\mathrm{S} \text {, from } \\
24 \text { to } 48 \text { hours. } \mathrm{SD}=\mathrm{S} \text {, } \\
\text { from } 1 \text { to } 3 \text { hours }\end{array}$ \\
\hline \multirow[t]{2}{*}{ Spence et al., $2011^{83}$} & 1 & $\begin{array}{l}\text { Shoulder } \\
\text { arthroscopy }\end{array}$ & Oral medication & Gabapentin & $\begin{array}{c}300 \mathrm{mg} \text { Gabapentin } 1 \text { hour } \\
\text { before surgery, then twice a } \\
\text { day for } 2 \text { days after surgery. } \\
\text { Interscalene nerve block also } \\
\text { used. }\end{array}$ & 26 military patients & $31.8 \pm 10.48$ & $\begin{array}{l}\text { NRS: Gabapentin = } \\
\text { Control, on days } 1 \\
\text { and } 2\end{array}$ & $\begin{array}{l}\text { Morphine Equivalents: } \\
\text { Gabapentin = Control, } \\
\text { on days } 1 \text { and } 2\end{array}$ \\
\hline & & & & Control & $\begin{array}{l}\text { Placebo } 1 \text { hour before } \\
\text { surgery, then twice a day for } \\
2 \text { days after surgery. } \\
\text { Interscalene nerve block also } \\
\text { used. }\end{array}$ & 31 military patients & $31.51 \pm 8.9$ & & \\
\hline
\end{tabular}




\begin{tabular}{|c|c|c|c|c|c|c|c|c|c|}
\hline $\begin{array}{l}\text { Author, } \\
\text { Publication } \\
\text { Year }\end{array}$ & $\begin{array}{l}\text { Level of } \\
\text { Evidence }\end{array}$ & Surgical Procedure & Intervention & Treatment Groups & Dosage & $\begin{array}{l}\text { Patients } \\
\qquad(n)\end{array}$ & $\begin{array}{c}\text { Age } \\
\text { (years) }\end{array}$ & $\begin{array}{l}\text { Post-Op Pain } \\
\text { Differences }\end{array}$ & $\begin{array}{l}\text { Post-Op Opioid } \\
\text { Consumption } \\
\text { Differences }\end{array}$ \\
\hline \multirow[t]{2}{*}{ Ahn et al., $2016^{27}$} & \multirow[t]{2}{*}{1} & \multirow{2}{*}{$\begin{array}{l}\text { Bankart repair } \\
(25 \%) \text { and rotator } \\
\text { cuff repair }(75 \%)\end{array}$} & \multirow[t]{2}{*}{ Oral medication } & Pregabalin & \multirow{2}{*}{$\begin{array}{c}\text { 1 150-mg Pregabalin capsule, } \\
1 \text { hour before anesthesia } \\
\text { induction } \\
\text { Placebo capsule, } 1 \text { hour } \\
\text { before anesthesia induction }\end{array}$} & 30 & $55 \pm 9$ & \multirow{2}{*}{$\begin{array}{c}\text { NRS: Pregabalin }< \\
\text { Control, from } 6 \text { to } 48 \\
\text { hours. Pregabalin }= \\
\text { Control, in PACU }\end{array}$} & $\begin{array}{c}\text { Fentanyl } \\
\text { Consumption: }\end{array}$ \\
\hline & & & & Control & & 30 & $51 \pm 12$ & & $\begin{array}{c}\text { Pregabalin }<\text { Control, } \\
0-6 \text { hours and } 0-48 \\
\text { hours. *Pregabalin }< \\
\text { Control, } 24-48 \text { hours. } \\
\text { Pregabalin = Control, } \\
\text { total consumption and } \\
\text { 6-24 hours }\end{array}$ \\
\hline \multirow[t]{2}{*}{$\begin{array}{l}\text { Mardani-Kivi et al., } \\
2016^{67}\end{array}$} & \multirow[t]{2}{*}{1} & \multirow[t]{2}{*}{ Bankart repair } & \multirow[t]{2}{*}{ Oral medication } & Gabapentin & $\begin{array}{c}1 \text { 600-mg Gabapentin } \\
\text { capsule, } 2 \text { hours before } \\
\text { surgery }\end{array}$ & 38 & $30.2 \pm 5.0$ & \multirow[t]{2}{*}{$\begin{array}{l}\text { VAS: Gabapentin }= \\
\text { Placebo }\end{array}$} & $\begin{array}{c}\text { Pethidine } \\
\text { Consumption: } \\
\text { Gabapentin < Placebo, }\end{array}$ \\
\hline & & & & Placebo & $\begin{array}{l}\text { Identical placebo capsule, } 2 \\
\text { hours before surgery }\end{array}$ & 38 & $28.3 \pm 4.4$ & & from 6 to 24 hours \\
\hline \multirow[t]{2}{*}{ Cho et al., $2011^{39}$} & \multirow[t]{2}{*}{1} & \multirow[t]{2}{*}{ Rotator cuff repair } & \multirow[t]{2}{*}{ Multimodal protocol } & Multimodal pain control & $\begin{array}{l}\text { Preoperative written and oral } \\
\text { education + pre-op } \\
\text { prophylactic oral } \\
\text { medication + intra-op } 50 \mathrm{~mL} \\
\text { cocktail of local analgesics }\end{array}$ & 40 & $57.6 \pm 8.2$ & \multirow{2}{*}{$\begin{array}{l}\text { VAS: Multimodal }= \\
\text { IV PCA, days } 1 \text { and } \\
\text { 2. Multimodal }<\text { IV } \\
\text { PCA, immediately } \\
\text { after surgery. } \\
{ }^{*} \text { Multimodal }<\text { IV } \\
\text { PCA, days 3-5 }\end{array}$} & \multirow[t]{2}{*}{$\begin{array}{c}\text { Analgesic } \\
\text { Consumption: } \\
\text { Multimodal }<\text { IV PCA } \\
\text { days } 0-5\end{array}$} \\
\hline & & & & $\begin{array}{l}\text { Intravenous patient- } \\
\text { controlled analgesia (IV } \\
\text { PCA) }\end{array}$ & $\begin{array}{l}\text { Individualized doses of } \\
\text { fentanyl, ketorolac, and } \\
\text { ondansteron } \mathrm{HCl}\end{array}$ & 30 & $55.1 \pm 7.5$ & & \\
\hline \multirow[t]{2}{*}{$\begin{array}{l}\text { Kraeutler et al., } \\
2015^{61}\end{array}$} & \multirow[t]{2}{*}{1} & \multirow{2}{*}{$\begin{array}{l}\text { All had rotator cuff } \\
\text { repair and/or } \\
\text { subacromial } \\
\text { decompression, } \\
\text { with distal clavicle } \\
\text { excision }(41 \%) \\
\text { and biceps } \\
\text { tenodesis }(37 \%) \text { as } \\
\text { most common } \\
\text { concomitant } \\
\text { procedures }\end{array}$} & \multirow[t]{2}{*}{$\begin{array}{l}\text { Nonpharmacological } \\
\text { intervention }\end{array}$} & $\begin{array}{c}\text { CC: Postoperative } \\
\text { compressive cryotherapy }\end{array}$ & $\begin{array}{c}\text { Used cryotherapy device } \\
\text { every other hour for days } 0-2 \\
\text { postsurgery, then } 2-3 \text { times } \\
\text { per day for an hour on days } \\
3-7 \text { postsurgery }\end{array}$ & 25 & 55.4 & \multirow[t]{2}{*}{$\begin{array}{c}\text { VAS: } C C=I W \text {, from } \\
4 \text { to } 6 \text { hours and on } \\
\text { days 1-7 }\end{array}$} & \multirow[t]{2}{*}{$\begin{array}{c}\text { Morphine Equivalents } \\
\mathrm{CC}=\mathrm{IW} \text {, from days } \\
1-7\end{array}$} \\
\hline & & & & $\begin{array}{l}\text { IW: Postoperative } \\
\text { standard ice wrap }\end{array}$ & $\begin{array}{l}\text { Used standard ice wrap every } \\
\text { other hour for days } 0-2 \\
\text { postsurgery, then } 2-3 \text { times } \\
\text { per day for an hour on days } \\
3-7 \text { postsurgery }\end{array}$ & 21 & 55.8 & & \\
\hline \multirow[t]{2}{*}{$\begin{array}{l}\text { Mahure et al., } \\
2017^{66}\end{array}$} & \multirow[t]{2}{*}{2} & \multirow[t]{2}{*}{$\begin{array}{l}\text { Rotator Cuff } \\
\text { Repair }\end{array}$} & \multirow[t]{2}{*}{$\begin{array}{c}\text { Non- } \\
\text { pharmacological } \\
\text { intervention }\end{array}$} & $\begin{array}{l}\text { Active transcutaneous } \\
\text { electrical nerve } \\
\text { stimulation (TENS) }\end{array}$ & \multirow[t]{2}{*}{$\begin{array}{l}\text { Continuous frequency of } 150 \\
\text { pps with pulse duration of } \\
150 \text { microseconds, active for } \\
30 \text { seconds then ramp down } \\
\text { for } 15 \text { seconds. Use TENS } \\
\text { unit } 4 \text { sessions/day, } 45 \\
\text { minutes/session, through } \\
\text { first postoperative week }\end{array}$} & 21 & $60.5 \pm 11.1$ & $\begin{array}{c}\text { VAS: TENS }< \\
\text { Placebo, from } 12 \text { to } \\
48 \text { hours, and from } \\
\text { days } 3 \text { to } 7\end{array}$ & $\begin{array}{l}\text { Percocet pills used: } \\
\text { TENS }<\text { Placebo, on } \\
\text { days } 2 \text { and } 7\end{array}$ \\
\hline & & & & Placebo TENS & & 16 & $56.4 \pm 12.2$ & \multirow{3}{*}{$\begin{array}{l}\text { VAS: *Pre-op } \\
\text { Education }<\text { No Pre- } \\
\text { op Education from } 2 \\
\text { to } 6 \text { weeks. Pre-op } \\
\text { Education }=\text { No Pre- } \\
\text { op Education, at } 3 \\
\text { months }\end{array}$} & \multirow{3}{*}{$\begin{array}{l}\text { Percocet pills used: } \\
\text { Pre-op education }<\text { No } \\
\text { Pre-op Education, } \\
\text { from } 6 \text { weeks to } 3 \\
\text { months. Pre-op } \\
\text { Education }=\text { No Pre-or } \\
\text { Education, at } 2 \text { weeks }\end{array}$} \\
\hline \multirow[t]{2}{*}{ Syed et al., $2018^{84}$} & \multirow[t]{2}{*}{1} & \multirow[t]{2}{*}{ Rotator cuff repair } & \multirow[t]{2}{*}{$\begin{array}{l}\text { Nonpharmacological } \\
\text { intervention }\end{array}$} & $\begin{array}{l}\text { No pre-op opioid } \\
\text { education }\end{array}$ & No video or handout & 66 & $58.0 \pm 9.4$ & & \\
\hline & & & & Pre-op opioid education & $\begin{array}{l}\text { 2-minute narrated video } \\
\text { with handout detailing the } \\
\text { risks of narcotic overuse and } \\
\text { abuse }\end{array}$ & 68 & $59.2 \pm 9.2$ & & \\
\hline
\end{tabular}

VAS, visual analog scale; NRS, numeric pain rating scale, DVPRS, defense and veterans pain rating scale, PACU, post-anesthesia care unit. 
showed significant differences in postoperative pain, while 19 studies also found significant differences in opioid consumption.

\section{ACL Reconstruction}

Five studies assessed the adductor canal block for ACL reconstruction. ${ }^{24,25,33,65,92}$ Three studies compared the adductor canal block to the femoral nerve block, ${ }^{24,65,33}$ with all three studies finding no difference in pain relief, and only Lynch et al. ${ }^{65}$ finding a difference in opioid consumption, with the adductor canal block allowing for less opioid consumption from 0 to 4 hours postoperatively. Meanwhile, Abdallah et al. ${ }^{25}$ used an adductor canal block with $20 \mathrm{~mL}$ of 1:1 ropivacaine and Lidocaine at three different locations: proximal, middle, and distal. They found that using the proximal block significantly decreases pain from 0 to 6 hours postoperatively, while dramatically decreasing opioid consumption from 0 to 24 hours as well. Lastly, Thapa et al. ${ }^{92}$ showed that an intermittent adductor canal block with $.5 \%$ ropivacaine provides similar pain relief, but dramatically decreased opioid consumption, relative to a continuous adductor canal block with the same dosage.

\section{Meniscectomy}

Three studies evaluated injections for meniscectomy. ${ }^{60,79,93}$ Sanel et al. $^{79}$ found that adding $20 \mathrm{mg}$ of tenoxicam to an intra-articular injection with .5\% bupivacaine dramatically decreases both pain at 12 hours postoperatively and opioid consumption at 24 hours postoperatively, as opposed to adding $2 \mathrm{mg}$ of morphine with the bupivacaine. However, pain was similar between the groups at 1, 2, 4, 6, and 24 hours postoperatively, so the dramatic difference in pain at 12 hours is not clear. Koltka et al. ${ }^{60}$ also evaluated intraarticular injections, but instead compared $500 \mathrm{mg}$ magnesium, $100 \mathrm{mg}$ of .5\% levobupivacaine, $8 \mathrm{mg}$ lornoxicam, and a saline placebo. All three intervention groups helped with pain relief and opioid consumption, with the only clear difference between intervention groups being that Lornoxicam decreased opioid consumption relative to magnesium from 0 to 24 hours postoperatively. Lastly, Lee et al. ${ }^{93}$ compared a control group with 2.5-, 5-, and 10- $\mu$ g dosages of hydromorphone added to bupivacaine intrathecal injections, finding that all the intervention groups provided better outcomes than the control group, but no significant differences between the hydromorphone dosage groups.

\section{Hip Arthroscopy}

Eight RCTs assessed pain and opioid consumption after hip arthroscopy (Table 4): 3 studies evaluated various types of nerve blocks, while 1 compared nerve block to intra-articular injection, 2 assessed localized injections, and 2 evaluated oral medication. Four RCTs isolated patients that underwent femoroacetabular impingement surgery. Four studies showed significant differences in postoperative pain, and 2 studies found significant differences in opioid consumption.

\section{Femoroacetabular Impingement}

Two studies evaluated nerve blocks for femoroacetabular impingement surgery. ${ }^{34,88}$ Behrends et al. ${ }^{34}$ found that a preoperative fascia iliaca block with $40 \mathrm{~mL}$ of $.2 \%$ bupivacaine provides similar pain relief and opioid consumption as a saline placebo. Xing et al. $^{88}$ found more encouraging pain management results, showing that a preoperative femoral nerve block with $20 \mathrm{~mL}$ of $.5 \%$ bupivacaine significantly decreases pain at several time points throughout the first postoperative week, and that opioid consumption also decreases from 1 to 2 days postoperatively, relative to a saline placebo. Unfortunately, the femoral nerve block also increased the rate of postoperative falls.

\section{Oral Medications}

Several studies show encouraging results for oral medications across shoulder, knee, and hip arthroscopy. For shoulder arthroscopy, Ahn et al. ${ }^{27}$ provided either a $150 \mathrm{mg}$ Pregabalin capsule or a placebo 1 hour before anesthesia induction (25\% Bankart repair, 75\% rotator cuff repair), and found that Pregabalin dramatically decreased pain from 6 to 48 hours postoperatively and opioid consumption from 0 to 48 hours postoperatively. Mardani-Kivi et al. ${ }^{67}$ instead provided either $600 \mathrm{mg}$ Gabapentin or a placebo to Bankart repair patients 2 hours before surgery, finding similar pain relief but significantly decreased opioid consumption from 6 to 24 hours postoperatively in the Gabapentin group. However, Spence et al. ${ }^{83}$ did not see a difference due to Gabapentin when provided to military patients preoperatively and postoperatively from shoulder arthroscopy, clouding the overall efficacy of Gabapentin. Across ACL reconstruction, meniscectomy, and knee arthroscopy, in general, providing either 120 $\mathrm{mg}$ Etoricoxib and $400 \mathrm{mg}$ Celecoxib preoperatively leads to significant improvements in both pain and

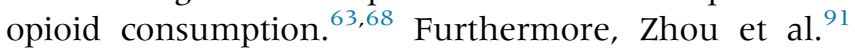
provided $400 \mathrm{mg}$ Celecoxib at various time points (1 day preoperatively, I hour preoperatively, and 4 hours postoperatively) to partial meniscectomy patients, finding that both the 1 day and 1 hour preoperative groups have better pain relief and slightly less opioid consumption relative to the 4 hours postoperative group. Lastly, in hip arthroscopy, $200 \mathrm{mg}$ Celecoxib given 1 hour before surgery may provide some pain relief and slightly decrease opioid consumption after femoroacetabular impingement surgery or labral repair; however, the effects are not strong and differ across studies. ${ }^{51,90}$ 
Table 3. Treatment Provided, Patient Population, Postoperative Pain, and Postoperative Opioid Consumption Summarized from all Included RCTs Regarding Knee Arthroscopy

\begin{tabular}{|c|c|c|c|c|c|c|c|c|c|}
\hline $\begin{array}{l}\text { Author, Publication } \\
\text { Year }\end{array}$ & $\begin{array}{l}\text { Level of } \\
\text { Evidence }\end{array}$ & Surgical Procedure & Intervention & Treatment Groups & Dosage & Patients $(n)$ & Age (years) & Post-Op Pain Differences & $\begin{array}{l}\text { Post-Op Opioid } \\
\text { Consumption } \\
\text { Differences }\end{array}$ \\
\hline \multirow{2}{*}{$\begin{array}{l}\text { Abdallah et al., } \\
2016^{24}\end{array}$} & \multirow[t]{2}{*}{1} & \multirow{2}{*}{$\begin{array}{l}\text { Unilateral ACL } \\
\text { Reconstruction }\end{array}$} & \multirow[t]{2}{*}{ Nerve block } & A: Adductor canal block & $20 \mathrm{~mL} .5 \%$ ropivacaine (with & 52 & 31.6 & \multirow{2}{*}{$\begin{array}{l}\text { VAS: } \mathrm{ACB}=\mathrm{FNB}, \text { from } \\
30 \mathrm{~min} \text { to } 24 \text { hours }\end{array}$} & \multirow{2}{*}{$\begin{array}{c}\text { Oral Morphine } \\
\text { Equivalents: ACB }= \\
\text { FNB, at } 24 \text { hours }\end{array}$} \\
\hline & & & & $\begin{array}{c}(\mathrm{ACB}) \\
\text { B: Femoral nerve block } \\
\text { (FNB) }\end{array}$ & $\begin{array}{c}\text { epinephrine) } \\
20 \mathrm{~mL} .5 \% \text { ropivacaine (with } \\
\text { epinephrine) }\end{array}$ & 48 & 33.3 & & \\
\hline \multirow[t]{3}{*}{ Abdallah et al., 2019} & \multirow[t]{3}{*}{1} & \multirow[t]{3}{*}{ ACL reconstruction } & \multirow[t]{3}{*}{ Nerve block } & $\begin{array}{l}\text { Proximal adductor canal } \\
\text { block }\end{array}$ & $\begin{array}{c}20 \mathrm{~mL} \text { of } 1: 1 \text { ropivacaine } .5 \% \\
\text { and Lidocaine } 2 \% \text { with epi } \\
1: 200,000\end{array}$ & 34 & 30 & \multirow{3}{*}{$\begin{array}{c}\text { VAS: *Proximal } \\
\text { Adductor Canal Block < } \\
\text { Mid }<\text { Distal, from } \\
\text { PACU-6 hr. All groups } \\
\text { are equal from } 12 \text { to } 24 \\
\text { hours }\end{array}$} & \multirow{3}{*}{$\begin{array}{c}\text { Total Morphine and } \\
\text { Morphine Equivalent } \\
\text { Consumed: Proximal } \\
\text { Adductor Canal Block } \\
\text { < Mid + Distal, until } \\
\text { discharge and from } 1 \\
\text { to } 24 \text { hours }\end{array}$} \\
\hline & & & & Mid adductor canal block & $\begin{array}{l}20 \mathrm{~mL} \text { of } 1: 1 \text { ropivacaine } .5 \% \\
\text { and Lidocaine } 2 \% \text { with epi }\end{array}$ & 38 & 31 & & \\
\hline & & & & Distal Canal Block & $\begin{array}{l}20 \mathrm{~mL} \text { of } 1: 1 \text { ropivacaine } .5 \% \\
\text { and Lidocaine } 2 \% \text { with epi }\end{array}$ & 36 & 29 & & \\
\hline \multirow[t]{2}{*}{ Thapa et al., $2016^{92}$} & \multirow[t]{2}{*}{1} & \multirow[t]{2}{*}{ ACL reconstruction } & \multirow[t]{2}{*}{ Nerve block } & $\begin{array}{c}\text { Continuous Adductor Canal } \\
\text { Block (ACB) }\end{array}$ & $.5 \%$ ropivacaine @ $2.5 \mathrm{~mL} / \mathrm{hr}$ & 25 & $25.2 \pm 6.4$ & \multirow{2}{*}{$\begin{array}{c}\text { VAS: Intermittent } \mathrm{ACB}< \\
\text { Continuous } \mathrm{ACP}, \text { from } 4 \\
\text { to } 12 \text { hours. Intermittent } \\
\mathrm{ACB}=\text { Continuous ACB, } \\
\text { at } 2 \text { and } 24 \text { hours }\end{array}$} & $\begin{array}{l}\text { Cumulative Oral } \\
\text { Morphine }\end{array}$ \\
\hline & & & & Intermittent ACB & $\begin{array}{c}.5 \% \text { ropivacaine, every } 6 \\
\text { hours }\end{array}$ & 25 & $27.7 \pm 7.2$ & & $\begin{array}{c}\text { Equivalents: } \\
\text { Intermittent ACB < } \\
\text { Continuous ACB, total } \\
\text { consumption }\end{array}$ \\
\hline \multirow[t]{2}{*}{ Lynch et al., $2019^{65}$} & \multirow[t]{2}{*}{1} & \multirow{2}{*}{$\begin{array}{l}\text { ACL Reconstruction, } \\
\text { with concomitant partial } \\
\text { meniscectomy }(35 \%) \text { or } \\
\text { meniscal repair }(10 \%)\end{array}$} & \multirow[t]{2}{*}{ Nerve block } & Adductor canal block (ACB) & $20 \mathrm{~mL} .5 \%$ ropivacaine & 30 & $21.2 \pm 4.2$ & \multirow{2}{*}{$\begin{array}{l}\text { VAS: } \mathrm{ACB}=\mathrm{FNB} \text {, from } \\
0 \text { hours to } 3 \text { days }\end{array}$} & Morphine Equivalents: \\
\hline & & & & Femoral nerve block (FNB) & $30 \mathrm{~mL} .5 \%$ ropivacaine & 30 & $21.5 \pm 5.4$ & & $\begin{array}{c}\mathrm{ACB}<\mathrm{FNB}, \text { from } 0 \text { to } 4 \\
\text { hours. } \mathrm{ACB}=\mathrm{FNB}, 4 \\
\text { hours to } 3 \text { days }\end{array}$ \\
\hline \multirow[t]{2}{*}{ Bailey et al., $2019^{33}$} & \multirow[t]{2}{*}{1} & $\begin{array}{l}\text { ACLR with patellar } \\
\text { tendon autograft, }\end{array}$ & \multirow[t]{2}{*}{ Nerve block } & $\begin{array}{l}\text { Femoral nerve blockade } \\
\text { (FNB) }\end{array}$ & $\begin{array}{l}30 \mathrm{~mL} \text { of } 0.2 \% \text { ropivacaine } \\
\text { with } 100 \mathrm{mcg} \text { clonidine }\end{array}$ & 38 & $24.4 \pm 8.8$ & \multirow[t]{2}{*}{ 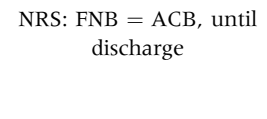 } & \multirow{2}{*}{$\begin{array}{c}\text { Morphine Equivalents: } \\
\text { FNB = ACB, until } \\
\text { discharge }\end{array}$} \\
\hline & & $\begin{array}{l}\text { concomitant meniscal } \\
\text { repair in } 78 \%\end{array}$ & & $\begin{array}{l}\text { Adductor canal nerve } \\
\text { blockade (ACB) }\end{array}$ & $\begin{array}{l}15 \mathrm{~mL} \text { of } 0.2 \% \text { ropivacaine } \\
\text { with } 100 \mathrm{mcg} \text { clonidine }\end{array}$ & 40 & $21.0 \pm 7.3$ & & \\
\hline \multirow[t]{2}{*}{$\begin{array}{l}\text { Espelund et al., } \\
\qquad 2014^{42}\end{array}$} & \multirow[t]{2}{*}{1} & \multirow[t]{2}{*}{$\begin{array}{l}\text { Minor arthroscopic knee } \\
\text { surgery }\end{array}$} & \multirow[t]{2}{*}{ Nerve block } & Ropivacaine & $\begin{array}{c}30 \mathrm{~mL} \text { of } 7.5 \mathrm{mg} / \mathrm{mL} \\
\text { ropivacaine }\end{array}$ & 36 & $46 \pm 14$ & \multirow{2}{*}{$\begin{array}{l}\text { VAS: } \\
\text { Ropivacaine }=\text { control, } \\
\text { from } 0 \text { to } 24 \text { hours }\end{array}$} & \multirow{2}{*}{$\begin{array}{c}\text { Total opioid } \\
\text { consumption: } \\
\text { Ropivacaine }<\text { control, } \\
\text { from } 0 \text { to } 2 \text { hours. } \\
\text { Ropivacaine }=\text { control, } \\
\text { from } 2 \text { to } 24 \text { hours. }\end{array}$} \\
\hline & & & & Control & $30 \mathrm{~mL}$ of isotonic saline & 35 & $43 \pm 14$ & & \\
\hline \multirow[t]{2}{*}{$\begin{array}{l}\text { Espelund et al., } \\
\qquad 2014^{43}\end{array}$} & 1 & $\begin{array}{c}50 \% \text { ACL } \\
\text { reconstruction, } 50 \% \\
\text { other major arthroscopic }\end{array}$ & Nerve block & Ropivacaine & $\begin{array}{l}30 \mathrm{~mL} \text { of } 7.5 \mathrm{mg} / \mathrm{mL} \\
\text { ropivacaine, } 30 \mathrm{~mL} \text { isotonic } \\
\text { saline } 45 \text { minutes later }\end{array}$ & 25 & $38 \pm 12$ & $\begin{array}{l}\text { VAS: Ropivacaine }< \\
\text { control, from } 15-45 \mathrm{~min} . \\
\text { Ropivacaine = control, }\end{array}$ & $\begin{array}{l}\text { Sufentanil consumption: } \\
\text { Ropivacaine = control, } \\
\text { from } 0 \text { to } 90 \mathrm{~min}\end{array}$ \\
\hline & & knee surgery & & Control & $\begin{array}{c}30 \mathrm{~mL} \text { of isotonic saline, } 30 \\
\mathrm{~mL} \text { of } 7.5 \mathrm{mg} / \mathrm{mL} \text { ropivacaine } \\
45 \mathrm{minutes} \text { later }\end{array}$ & 25 & $34 \pm 14$ & from 60-90 min & \\
\hline Hanson et al., $2013^{46}$ & 1 & Medial meniscectomy & Nerve block & $\begin{array}{l}\text { ACB: Ultrasound-guided } \\
\text { adductor canal block }\end{array}$ & $\begin{array}{l}15 \mathrm{~mL} \text { of } .5 \% \text { ropivacaine } \\
\text { with 1:400,000 epinephrine }\end{array}$ & 24 & $54 \pm 11$ & $\begin{array}{l}\text { NRS: ACB }<\text { sham, in } \\
\text { PACU, at discharge, and }\end{array}$ & $\begin{array}{c}\text { Oral Morphine } \\
\text { Equivalents: } \mathrm{ACB}<\end{array}$ \\
\hline & & & & $\begin{array}{l}\text { Sham: Ultrasound-guided } \\
\text { sham injection }\end{array}$ & $2 \mathrm{~mL}$ normal saline & 24 & $51 \pm 11$ & $\begin{array}{c}\text { from } 12 \text { to } 24 \mathrm{hr} \text {. } \mathrm{ACB}= \\
\text { sham, at } 6 \text { hours }\end{array}$ & Sham, over 24 hours \\
\hline Hsu et al., $2013^{48}$ & 1 & $\begin{array}{c}87 \% \text { soft-tissue } \\
\text { (meniscectomy, meniscal }\end{array}$ & Nerve block & $\begin{array}{l}\text { INF: Block of infrapatellar } \\
\text { branch of saphenous nerve }\end{array}$ & $10 \mathrm{~mL}$ of $.25 \%$ bupivacaine & 33 & $51.7 \pm 12.1$ & $\begin{array}{l}\text { NRS: INF < Placebo, } \\
\text { immediately }\end{array}$ & $\begin{array}{l}\text { IV Ketorolac, } \\
\text { Hydrocodone, and }\end{array}$ \\
\hline & & $\begin{array}{l}\text { repair), } 26 \% \text { single } \\
\text { osseous, } 24 \% \text { multiple } \\
\text { osseous procedures }\end{array}$ & & Placebo & Saline solution & 32 & $49.6 \pm 14.1$ & $\begin{array}{c}\text { postoperatively, at } 1 \\
\text { hour, and on arrival at } \\
\text { home. INF = Placebo, } \\
\text { from } 2 \text { to } 4 \text { hours } \\
\text { postoperatively, and 0- } \\
24 \text { hours after arriving } \\
\text { home }\end{array}$ & $\begin{array}{c}\text { Fentanyl, oral } \\
\text { Hydrocodone, and Total } \\
\text { Oral Morphine } \\
\text { Equivalents: INF }= \\
\text { Placebo, } 0-48 \text { hours }\end{array}$ \\
\hline
\end{tabular}




\begin{tabular}{|c|c|c|c|c|c|c|c|c|c|}
\hline $\begin{array}{l}\text { Author, Publication } \\
\text { Year }\end{array}$ & $\begin{array}{l}\text { Level of } \\
\text { Evidence }\end{array}$ & Surgical Procedure & Intervention & Treatment Groups & Dosage & Patients $(n)$ & Age (years) & Post-Op Pain Differences & $\begin{array}{l}\text { Post-Op Opioid } \\
\text { Consumption } \\
\text { Differences }\end{array}$ \\
\hline \multirow[t]{2}{*}{$\begin{array}{l}\text { Westergaard et al., } \\
2014^{86}\end{array}$} & \multirow[t]{2}{*}{1} & \multirow[t]{2}{*}{$\begin{array}{l}61 \% \text { synovectomy, } 53 \% \\
\text { meniscectomy, } 37 \% \\
\text { chondrosectomy, with } \\
\text { other concomitant } \\
\text { procedures }\end{array}$} & \multirow[t]{2}{*}{ Nerve block } & Ropivacaine & $\begin{array}{l}20 \mathrm{~mL} \text { of } .75 \% \text { ropivacaine } \\
\text { prepared }-7.5 \mathrm{~mL} \text { around } \\
\text { the saphenous nerve and } 7.5 \\
\mathrm{~mL} \text { around the posterior } \\
\text { branch of obturator nerve }\end{array}$ & 29 & 31 & $\begin{array}{l}\text { NRS: ropivacaine }= \\
\text { Saline, from } 0 \text { to } 24 \\
\text { hours }\end{array}$ & $\begin{array}{l}\text { Morphine consumed: } \\
\text { ropivacaine = Saline, } \\
\text { from } 0 \text { to } 24 \text { hours }\end{array}$ \\
\hline & & & & Saline & $\begin{array}{l}20 \mathrm{~mL} \text { of isotonic saline } \\
\text { prepared }-7.5 \mathrm{~mL} \text { around } \\
\text { the saphenous nerve and } 7.5 \\
\mathrm{~mL} \text { around the posterior } \\
\text { branch of obturator nerve }\end{array}$ & 30 & 42 & & \\
\hline \multirow{2}{*}{$\begin{array}{l}\text { Marinković et al., } \\
2016^{69}\end{array}$} & \multirow[t]{2}{*}{1} & \multirow[t]{2}{*}{ Knee arthroscopy } & \multirow{2}{*}{$\begin{array}{l}\text { Peripheral nerve } \\
\text { block }\end{array}$} & GA: General anesthesia & - & 30 children & $13.5 \pm 3.2$ & Wong Baker Faces & $\begin{array}{c}\text { Morphine } \\
\text { a }\end{array}$ \\
\hline & & & & $\begin{array}{l}\text { PNB +GA: Peripheral nerve } \\
\text { block + general anesthesia/ } \\
\text { sedation }\end{array}$ & $\begin{array}{c}1 \mathrm{~mL} / \mathrm{kg} \text { of } .25 \% \text { or. } 33 \% \\
\text { levobupivacaine } \\
\text { administered with } \\
\text { ultrasound guidance. } 43 \% \\
\text { femoral, obturator, ischiatic } \\
\text { block; } 33 \% \text { femoral, } \\
\text { obturator block; } 23 \% \\
\text { femoral, ischiatic block }\end{array}$ & 30 children & $15.2 \pm 1.6$ & $\begin{array}{l}\text { Scale: PNB }+ \text { GA }< \\
\text { GA, from } 2 \text { to } 12 \text { hours }\end{array}$ & $\begin{array}{c}\text { Equivalents: PNB + } \\
\text { GA < GA, until } \\
\text { discharge }\end{array}$ \\
\hline \multirow[t]{2}{*}{ Keller et al., $2019^{56}$} & \multirow[t]{2}{*}{1} & \multirow[t]{2}{*}{ ACL reconstruction } & \multirow{2}{*}{$\begin{array}{l}\text { Nerve block vs nerve } \\
\text { block + local } \\
\text { injection }\end{array}$} & Femoral Nerve Block (FNB) & $\begin{array}{c}20 \mathrm{~mL} .5 \% \text { Bupivacaine } \\
\text { without epi }\end{array}$ & 21 & 35.1 & \multirow{2}{*}{$\begin{array}{c}\text { VAS: *FNB }+\mathrm{PCI}<\mathrm{FNB} \text {, } \\
\text { at until discharge and at } \\
1 \text { hour. FNB }+\mathrm{PCI}= \\
\text { FNB, at } 20 \text { min and days } \\
1-4\end{array}$} & \multirow{2}{*}{$\begin{array}{c}\text { Number of Vicodin Pills } \\
\text { Used: FNB }+ \text { PCI }<\text { FNB } \\
\text { on day } 4\end{array}$} \\
\hline & & & & $\begin{array}{l}\text { FNB + Posterior capsule } \\
\text { injection (PCI) }\end{array}$ & $\begin{array}{l}\text { FNB }+20 \mathrm{~mL} .5 \% \\
\text { Bupivacaine without epi in } \\
\text { posterior capsule, injected } \\
\text { before drilling femoral tunnel }\end{array}$ & 21 & 32.5 & & \\
\hline \multirow[t]{2}{*}{$\begin{array}{l}\text { Moyano et al., } \\
2016^{73}\end{array}$} & \multirow[t]{2}{*}{1} & \multirow[t]{2}{*}{$\begin{array}{c}35 \% \text { ACL repair, } 28 \% \\
\text { multiple procedures, } \\
24 \% \text { meniscectomy, } \\
13 \% \text { other }\end{array}$} & \multirow[t]{2}{*}{ IV injection } & DM: Dexamethasone & $\begin{array}{c}2 \mathrm{~mL} \text { of a } 5 \mathrm{mg} / \mathrm{mL} \\
\text { dexamethasone phosphate } \\
\text { solution, during anesthetic } \\
\text { induction }\end{array}$ & 37 & 39.9 & \multirow[t]{2}{*}{$\begin{array}{l}\text { VAS: DM }>S \text {, at } 4 \text { hours. } \\
\text { DM }=S \text {, in PACU, and at } \\
8 \text { and } 12 \text { hours }\end{array}$} & \multirow[t]{2}{*}{$\begin{array}{l}\text { Number of Codeine } \\
\text { Tablets Taken: DM = S, } \\
\text { from } 0 \text { to } 48 \text { hours }\end{array}$} \\
\hline & & & & S: Saline & $\begin{array}{l}2 \mathrm{~mL} \text { of } .9 \% \text { normal saline, } \\
\text { during anesthetic induction }\end{array}$ & 41 & 44.3 & & \\
\hline \multirow[t]{3}{*}{ Amin et al., $2011^{29}$} & \multirow[t]{3}{*}{2} & \multirow[t]{3}{*}{ ACL reconstruction } & \multirow[t]{3}{*}{$\begin{array}{c}\text { Intraarticular } \\
\text { patient-controlled } \\
\text { analgesia (PCA) }\end{array}$} & $\begin{array}{l}\text { RMX: Morphine + } \\
\text { ropivacaine + xefocam } \\
\text { mixture }\end{array}$ & $\begin{array}{l}\text { PCA of . } 25 \% \text { ropivacaine, } .2 \\
\mathrm{mg} / \mathrm{mL} \text { morphine, } 1 \mathrm{mg} / \mathrm{mL} \\
\text { xefocam (Lornoxicam) }\end{array}$ & 15 & $32 \pm 3$ & \multirow{3}{*}{$\begin{array}{c}\text { VAS: } \mathrm{RMX}=\mathrm{RM}=\mathrm{C} \text {, at } \\
4 \text { hr. } \mathrm{RMX}<\mathrm{RM}+\mathrm{C} \text {, at } \\
\text { 8-16 hours. } \mathrm{RMX}<\mathrm{RM} \\
\quad<\mathrm{C} \text {, at } 24 \text { hours }\end{array}$} & \multirow[t]{3}{*}{$\begin{array}{l}\text { Rescue IV Morphine: } \\
\text { RMX }<\text { RM, at } 24 \text { hours } \\
\text { *RM }<\text { C, at } 24 \text { hours }\end{array}$} \\
\hline & & & & $\begin{array}{l}\text { RM: Ropivacaine + } \\
\text { morphine mixture }\end{array}$ & $\begin{array}{c}\text { PCA of } .25 \% \text { ropivacaine, } .2 \\
\mathrm{mg} / \mathrm{mL} \text { morphine }\end{array}$ & 15 & $27 \pm 3$ & & \\
\hline & & & & Control: No drug & - & 15 & $35 \pm 3$ & & \\
\hline \multirow[t]{2}{*}{ Sanel et al., $2016^{79}$} & \multirow[t]{2}{*}{1} & \multirow[t]{2}{*}{$\begin{array}{l}\text { Isolated partial } \\
\text { meniscectomy }\end{array}$} & \multirow[t]{2}{*}{$\begin{array}{l}\text { Intra-articular } \\
\text { injection }\end{array}$} & $\begin{array}{l}\text { TEN: tenoxicam with } \\
\text { bupivacaine }\end{array}$ & $\begin{array}{l}22 \mathrm{~mL} \text { of } .5 \% \text { bupivacaine } \\
100 \mathrm{mg}+\text { tenoxicam } 20 \mathrm{mg}, \\
\text { after the surgery and before } \\
\text { tourniquet deflation }\end{array}$ & 120 & 36 & \multirow[t]{2}{*}{$\begin{array}{l}\text { VAS and NRS: TEN < } \\
\text { MOR, at } 12 \text { hours. } \\
\text { TEN = MOR, at } 1,2,4,6, \\
\text { and } 24 \text { hours }\end{array}$} & \multirow[t]{2}{*}{$\begin{array}{l}\text { Total Analgesic: TEN } \\
\text { MOR, at } 24 \text { hours }\end{array}$} \\
\hline & & & & $\begin{array}{l}\text { MOR: morphine with } \\
\text { bupivacaine }\end{array}$ & $\begin{array}{l}22 \mathrm{~mL} \text { of } .5 \% \text { bupivacaine } \\
100 \mathrm{mg}+\text { morphine } 2 \mathrm{mg}, \\
\text { after the surgery and before } \\
\text { tourniquet deflation }\end{array}$ & 120 & 40 & & \\
\hline
\end{tabular}




\begin{tabular}{|c|c|c|c|c|c|c|c|c|c|}
\hline $\begin{array}{c}\text { Author, Publication } \\
\text { Year }\end{array}$ & $\begin{array}{l}\text { Level of } \\
\text { Evidence }\end{array}$ & Surgical Procedure & Intervention & Treatment Groups & Dosage & Patients $(n)$ & Age (years) & Post-Op Pain Differences & $\begin{array}{l}\text { Post-Op Opioid } \\
\text { Consumption } \\
\text { Differences }\end{array}$ \\
\hline \multirow[t]{5}{*}{$\begin{array}{l}\text { Arti and } \\
\text { Mehdinasab, } \\
2011^{30}\end{array}$} & \multirow[t]{5}{*}{1} & \multirow[t]{5}{*}{ ACL reconstruction } & \multirow[t]{5}{*}{$\begin{array}{l}\text { Intra-articular } \\
\text { injection }\end{array}$} & Morphine & $\begin{array}{c}\text { At end of procedure: } 9.5 \mathrm{~mL} \\
\text { bupivacaine }+5 \mathrm{mg} \\
\text { morphine }\end{array}$ & 30 & $31.5 \pm 5.9$ & \multirow{5}{*}{$\begin{array}{l}\text { VAS: Morphine }<\text { all } \\
\text { other groups, } 0-12 \text { hours } \\
\text { after surgery. All other } \\
\text { groups }<\text { Placebo, } 0-12 \\
\text { hours after surgery }\end{array}$} & \multirow{5}{*}{$\begin{array}{c}\text { Morphine Equivalents: } \\
\text { Morphine + Methadone } \\
<\text { Pethidine + Tramadol } \\
\text { < Placebo, 0-12 hours } \\
\text { after surgery }\end{array}$} \\
\hline & & & & Methadone & $\begin{array}{c}\text { At end of procedure: } 9.5 \mathrm{~mL} \\
\text { bupivacaine }+5 \mathrm{mg} \\
\text { methadone }\end{array}$ & 30 & $28.9 \pm 7.6$ & & \\
\hline & & & & Pethidine & $\begin{array}{c}\text { At end of procedure: } 9.5 \mathrm{~mL} \\
\text { bupivacaine }+37.5 \mathrm{mg} \\
\text { pethidine }\end{array}$ & 30 & $26.8 \pm 7.8$ & & \\
\hline & & & & Tramadol & $\begin{array}{l}\text { At end of procedure: } 9.5 \mathrm{~mL} \\
\text { bupivacaine }+100 \mathrm{mg} \\
\text { tramadol }\end{array}$ & 30 & $27.5 \pm 7.4$ & & \\
\hline & & & & Placebo & $\begin{array}{l}\text { At end of procedure: } 9.5 \mathrm{~mL} \\
\text { bupivacaine }+.5 \mathrm{~mL} \text { normal } \\
\text { saline }\end{array}$ & 30 & $28.6 \pm 5.3$ & & \\
\hline \multirow[t]{3}{*}{ Mitra et al., $2011^{72}$} & \multirow[t]{3}{*}{1} & \multirow[t]{3}{*}{$\begin{array}{c}70 \% \text { ACL repair, } 20 \% \\
\text { diagnostic arthroscopy, } \\
10 \% \text { other }\end{array}$} & \multirow[t]{3}{*}{$\begin{array}{l}\text { Intra-articular } \\
\text { injection }\end{array}$} & Tramadol & $\begin{array}{c}30 \mathrm{~mL} .25 \% \text { bupivacaine }+1 \\
\mathrm{~mL}(50 \mathrm{mg} \text { ) tramadol, at end } \\
\text { of surgery }\end{array}$ & 20 & $31.65 \pm 12.86$ & \multirow{3}{*}{$\begin{array}{l}\text { VAS: Fentanyl < } \\
\text { Tramadol + Saline, } \\
\text { from } \mathbf{0} \text { to } \mathbf{8} \mathbf{h r} \text {. } \\
\text { *Tramadol < Saline, } \\
\text { from } 0 \text { to } 8 \text { hours }\end{array}$} & \multirow[t]{3}{*}{$\begin{array}{l}\text { Total Analgesic: } \\
\text { Fentanyl }+ \text { Tramadol }< \\
\text { Saline, from } 0 \text { to } 8 \text { hours }\end{array}$} \\
\hline & & & & Fentanyl & $\begin{array}{c}30 \mathrm{~mL} .25 \% \text { bupivacaine }+1 \\
\text { ML (50 } \mu \mathrm{g} \text { ) fentanyl, at end } \\
\text { of surgery }\end{array}$ & 20 & $26.55 \pm 8.02$ & & \\
\hline & & & & Saline & $\begin{array}{l}30 \mathrm{~mL} .5 \% \text { bupivacaine }+1 \\
\mathrm{~mL} \text { normal saline, at end of } \\
\text { surgery }\end{array}$ & 20 & $28.05 \pm 10.76$ & & \\
\hline \multirow[t]{3}{*}{ Kager et al., $2011^{50}$} & \multirow[t]{3}{*}{2} & \multirow{3}{*}{$\begin{array}{l}75 \% \text { meniscus resection } \\
\text { and cartilage smoothing, } \\
19 \% \text { cartilage smoothing } \\
\text { only, and } 6 \% \text { cruciate } \\
\text { ligament repair }\end{array}$} & \multirow[t]{3}{*}{$\begin{array}{l}\text { Intra-articular } \\
\text { injection }\end{array}$} & $5 \mathrm{mg}$ labetalol & $\begin{array}{c}20 \mathrm{~mL} \text { intra-articularly with } \\
5 \mathrm{mg} \text { labetalol, at end of } \\
\text { surgery }\end{array}$ & 21 & $48.0 \pm 3.5$ & \multirow[t]{3}{*}{$\begin{array}{l}\text { VAS and VRS: } 5 \mathrm{mg}= \\
2.5 \mathrm{mg}=\text { Placebo, from } \\
30 \mathrm{~min} \text { to } 24 \text { hours }\end{array}$} & \multirow{3}{*}{$\begin{array}{l}\text { Morphine Consumption: } \\
\text { Placebo }+5 \mathrm{mg}<2.5 \\
\mathrm{mg} \text {, from } 30 \mathrm{~min} \text { to } 24 \\
\text { hours. Placebo }<5 \mathrm{mg} \text {, } \\
\text { from } 30 \mathrm{~min} \text { to } 1 \text { hour, } \\
\text { and from } 4 \text { to } 24 \text { hours. } \\
\text { Placebo }=5 \mathrm{mg} \text {, from } 2 \\
\text { to } 3 \text { hours }\end{array}$} \\
\hline & & & & $2.5 \mathrm{mg}$ labetalol & $\begin{array}{l}20 \mathrm{~mL} \text { intra-articularly with } \\
2.5 \mathrm{mg} \text { labetalol, at end of } \\
\text { surgery }\end{array}$ & 18 & $41.4 \pm 3.9$ & & \\
\hline & & & & Placebo & $\begin{array}{l}20 \mathrm{~mL} \text { intra-articularly with } \\
\text { normal saline, at end of } \\
\text { surgery }\end{array}$ & 24 & $49.0 \pm 2.5$ & & \\
\hline \multirow[t]{4}{*}{ Koltka et al., $2011^{60}$} & \multirow[t]{4}{*}{1} & \multirow[t]{4}{*}{ Meniscectomy } & \multirow[t]{4}{*}{$\begin{array}{l}\text { Intra-articular } \\
\text { injection }\end{array}$} & Magnesium & $\begin{array}{l}500 \mathrm{mg} \text { magnesium sulfate } \\
\text { intra-articularly in } 20 \mathrm{~mL} \\
\text { saline, before tourniquet } \\
\text { deflation }\end{array}$ & 30 & $48.4 \pm 11$ & \multirow{4}{*}{$\begin{array}{c}\text { NRS: All groups are } \\
\text { equal at } 1 \text { hour } \\
\text { *Lornoxicam }<\text { Placebo, } \\
\text { at } 2 \text { hours. } \\
\text { Levobupivacaine }< \\
\text { Placebo, at } 2 \text { hours. } \\
\text { Magnesium = Placebo, at } \\
2 \text { hours. Magnesium + } \\
\text { Levobupivacaine }+ \\
\text { Lornoxicam < Placebo, } \\
\text { at } 4 \text { hours. All groups } \\
\text { are equal from } 12 \text { to } 48 \\
\text { hours }\end{array}$} & $\begin{array}{l}\text { Tramadol Consumption } \\
\text { and Number of Pills } \\
\text { Consumed: *Lornoxicam } \\
\text { < Placebo, from } 0 \text { to } 4\end{array}$ \\
\hline & & & & Levobupivacaine & $\begin{array}{c}100 \mathrm{mg} \text { levobupivacaine } \\
(.5 \%) \text { of } 20 \mathrm{~mL} \text { local } \\
\text { anesthetic, before tourniquet } \\
\text { deflation }\end{array}$ & 30 & $50.6 \pm 12$ & & $\begin{array}{c}\text { hr. Magnesium }+ \\
\text { Levobupivacaine }< \\
\text { Placebo, from } 0 \text { to } 4 \\
\text { hr. *Lornoxicam }<\end{array}$ \\
\hline & & & & Lornoxicam & $\begin{array}{l}8 \mathrm{mg} \text { lornoxicam intra- } \\
\text { articularly in } 20 \mathrm{~mL} \text { saline, } \\
\text { before tourniquet deflation }\end{array}$ & 30 & $42.5 \pm 9.7$ & & $\begin{array}{l}\text { Placebo, from } 0 \text { to } 48 \mathrm{hr} \text {. } \\
\text { Magnesium }= \\
\text { Levobupivacaine }=\end{array}$ \\
\hline & & & & Placebo & $\begin{array}{l}20 \mathrm{~mL} \text { saline intra- } \\
\text { articularly, before tourniquet } \\
\text { deflation }\end{array}$ & 30 & $46 \pm 15.6$ & & $\begin{array}{c}\text { Placebo, from } 0 \text { to } 48 \\
\text { hours. Lornoxicam }< \\
\text { Magnesium, from } 0 \text { to } 24 \\
\text { hours }\end{array}$ \\
\hline
\end{tabular}




\begin{tabular}{|c|c|c|c|c|c|c|c|c|c|}
\hline $\begin{array}{l}\text { Author, Publication } \\
\text { Year }\end{array}$ & $\begin{array}{l}\text { Level of } \\
\text { Evidence }\end{array}$ & Surgical Procedure & Intervention & Treatment Groups & Dosage & Patients $(n)$ & Age (years) & Post-Op Pain Differences & $\begin{array}{l}\text { Post-Op Opioid } \\
\text { Consumption } \\
\text { Differences }\end{array}$ \\
\hline \multirow[t]{4}{*}{ Lee et al., $2012^{93}$} & \multirow[t]{4}{*}{2} & \multirow[t]{4}{*}{ Partial meniscectomy } & \multirow[t]{4}{*}{ Intrathecal injection } & $\begin{array}{c}10 \mathrm{HM}: 10 \mu \mathrm{g} \\
\text { hydromorphone }\end{array}$ & $\begin{array}{l}\text { Spinal anesthesia with: } 10 \mu \mathrm{g} \\
\text { hydromorphone }+1.2 \mathrm{~mL} \text { ( } 6 \\
\mathrm{mg} \text { ) of } .5 \% \text { hyperbaric } \\
\text { bupivacaine, in } .05 \mathrm{~mL} \\
\text { isotonic saline, prior to } \\
\text { surgery }\end{array}$ & 15 & $36.3 \pm 12.3$ & \multirow{4}{*}{$\begin{array}{c}\text { VAS: All groups are } \\
\text { equal, at } 30 \mathrm{~min} \text { and } 2 \\
\text { hours } 2.5 \mathrm{HM}, 5 \mathrm{HM} \text {, } \\
\text { and } 10 \mathrm{HM}<\mathrm{Control} \text {, } \\
\text { from } 4-6 \text { hours. } 5 \mathrm{HM} \\
\text { and } 10 \mathrm{HM}<\mathrm{Control} \text {, at } \\
12 \text { hours. } 2.5 \mathrm{HM}= \\
\text { Control, at } 12 \text { hours. All } \\
\text { groups are equal, at } 24 \\
\text { hours }\end{array}$} & \multirow{4}{*}{$\begin{array}{c}\text { Number of required } \\
\text { analgesic injections: } 5 \\
\text { HM and } 10 \mathrm{HM}< \\
\text { Control and } 2.5 \mathrm{HM}, 0- \\
24 \text { hours } \\
\end{array}$} \\
\hline & & & & $5 \mathrm{HM}: 5 \mu \mathrm{g}$ hydromorphone & $\begin{array}{l}\text { Spinal anesthesia with: } 5 \mu \mathrm{g} \\
\text { hydromorphone }+1.2 \mathrm{~mL} \text { ( } 6 \\
\mathrm{mg} \text { ) of } .5 \% \text { hyperbaric } \\
\text { bupivacaine, in } .05 \mathrm{~mL} \\
\text { isotonic saline, prior to } \\
\text { surgery }\end{array}$ & 15 & $36.5 \pm 15.1$ & & \\
\hline & & & & $\begin{array}{l}2.5 \mathrm{HM}: 2.5 \mu \mathrm{g} \\
\text { hydromorphone }\end{array}$ & $\begin{array}{l}\text { Spinal anesthesia with: } 2.5 \\
\mu \mathrm{g} \text { hydromorphone }+1.2 \mathrm{~mL} \\
\text { (6 mg) of } .5 \% \text { hyperbaric } \\
\text { bupivacaine, in } .05 \mathrm{~mL} \\
\text { isotonic saline, prior to } \\
\text { surgery }\end{array}$ & 15 & $38.9 \pm 12.4$ & & \\
\hline & & & & Control & $\begin{array}{l}\text { Spinal anesthesia with: } 6 \mathrm{mg} \\
\text { hyperbaric bupivacaine in } .05 \\
\mathrm{~mL} \text { isotonic saline, prior to } \\
\text { surgery }\end{array}$ & 15 & $39.9 \pm 13.7$ & & \\
\hline \multirow[t]{4}{*}{ Sayin et al., $2015^{76}$} & \multirow[t]{4}{*}{1} & \multirow{4}{*}{$\begin{array}{l}\text { Meniscopathic knee } \\
\text { surgery }\end{array}$} & \multirow[t]{4}{*}{ Local anesthesia } & C: Control & No description & 20 & $30.2 \pm 6.8$ & \multirow{4}{*}{$\begin{array}{l}\text { VAS: }{ }^{*} \mathrm{~L}+\mathrm{T} \text { and } \mathrm{L}+\mathrm{F}< \\
\mathrm{C} \text { and } \mathrm{L} \text {, from } 1 \text { to } 24 \\
\text { hours. Also }{ }^{*} \mathrm{~L}+\mathrm{T}<\mathrm{L}+ \\
\text { F, from } 2 \text { to } 4 \text { hours }\end{array}$} & \multirow{4}{*}{$\begin{array}{l}\text { Number of times needing } \\
\text { post-op Analgesia: } \mathrm{L}+\mathrm{T} \\
\text { and } \mathrm{L}+\mathrm{F}<\mathrm{C} \text { and } \mathrm{L} \text {, } \\
\text { total doses }\end{array}$} \\
\hline & & & & L: Levobupivacaine & $\begin{array}{l}20 \mathrm{~mL} \text { of Levobupivacaine } \\
2.5 \mathrm{mg} / \mathrm{mL}, 7 \mathrm{~mL} \text { before } \\
\text { surgery and } 13 \mathrm{~mL} \text { at the end } \\
\text { of surgery }\end{array}$ & 20 & $32.6 \pm 7.0$ & & \\
\hline & & & & $\begin{array}{l}\mathrm{L}+\mathrm{T}: \text { Levobupivacaine }+ \\
\text { Tramadol }\end{array}$ & $\begin{array}{c}20 \mathrm{~mL} \text { of Levobupivacaine } \\
2.5 \mathrm{mg} / \mathrm{mL}+50 \mathrm{mg} \\
\text { Tramadol, } 7 \mathrm{~mL} \text { before } \\
\text { surgery and } 13 \mathrm{~mL} \text { at the end } \\
\text { of surgery }\end{array}$ & 20 & $36.2 \pm 8.8$ & & \\
\hline & & & & $\begin{array}{l}\text { L+F: Levobupivacaine }+ \\
\text { Fentanyl }\end{array}$ & $\begin{array}{l}20 \mathrm{~mL} \text { of Levobupivacaine } \\
2.5 \mathrm{mg} / \mathrm{mL}+50 \mathrm{mcg} \\
\text { Fentanyl, } 7 \mathrm{~mL} \text { before } \\
\text { surgery and } 13 \mathrm{~mL} \text { at the end } \\
\text { of surgery }\end{array}$ & 20 & $34.7 \pm 10.2$ & & \\
\hline \multirow[t]{2}{*}{$\begin{array}{l}\text { Premkumar et al., } \\
2016^{98}\end{array}$} & \multirow[t]{2}{*}{2} & \multirow[t]{2}{*}{$\begin{array}{l}\text { ACL reconstruction with } \\
\text { quadriceps autograft }\end{array}$} & \multirow[t]{2}{*}{$\begin{array}{l}\text { Surgical site } \\
\text { injection }\end{array}$} & $\begin{array}{l}\text { Local liposomal bupivacaine } \\
\text { (LLB) }\end{array}$ & $\begin{array}{c}40 \mathrm{~mL} \text { suspension of } 20 \mathrm{~mL} \\
\text { liposomal bupivacaine }+20 \\
\text { mL Saline, } 30 \mathrm{~mL} \text { injected } \\
\text { into graft harvest site and } 10 \\
\mathrm{~mL} \text { into superficial skin }\end{array}$ & 14 & & \multirow[t]{2}{*}{$\begin{array}{l}\text { NRS: LLB }=\text { LB, from } \\
\text { PACU until day } 6\end{array}$} & \multirow[t]{2}{*}{$\begin{array}{c}\text { PACI IV } \\
\text { Hydromorphone, PACE } \\
\text { Fentanyl, and PACE } \\
\text { Oxycodone Equivalents } \\
\text { Consumed: LLB }=\text { LB, } \\
\text { from PACU until day } 6\end{array}$} \\
\hline & & & & Local bupivacaine (LB) & $\begin{array}{c}40 \mathrm{~mL} \text { suspension of } 20 \mathrm{~mL} \\
\text { bupivacaine }+20 \mathrm{~mL} \text { Saline, } \\
30 \mathrm{~mL} \text { injected into graft } \\
\text { harvest site and } 10 \mathrm{~mL} \text { into } \\
\text { superficial skin }\end{array}$ & 15 & & & \\
\hline \multirow[t]{3}{*}{ Zhou et al., $2017^{91}$} & \multirow[t]{3}{*}{1} & \multirow[t]{3}{*}{ Partial Meniscectomy } & \multirow[t]{3}{*}{ Oral medication } & Celecoxib 4 hours post-op & 400 mg Celecoxib & 60 & $35.9 \pm 6.6$ & \multirow{3}{*}{$\begin{array}{l}\text { VAS: Very Early + Early } \\
\text { < Post op Celecoxib, } \\
\text { from } 4 \text { to } 36 \text { hours }\end{array}$} & \multirow{3}{*}{$\begin{array}{c}\text { Rescue analgesic } \\
\text { consumed: Very Early }+ \\
\text { Early almost }<\text { Post-op } \\
\text { Celecoxib }(P=.06) \text {, from } \\
24 \text { to } 48 \text { hours }\end{array}$} \\
\hline & & & & & $400 \mathrm{mg}$ Celecoxib & 62 & $36.0 \pm 6.1$ & & \\
\hline & & & & $\begin{array}{l}\text { Celecoxib } 1 \text { Day Pre-op (very } \\
\text { early) }\end{array}$ & $400 \mathrm{mg}$ Celecoxib & 60 & $34.7 \pm 7.1$ & & \\
\hline
\end{tabular}




\begin{tabular}{|c|c|c|c|c|c|c|c|c|c|}
\hline $\begin{array}{c}\text { Author, Publication } \\
\text { Year }\end{array}$ & $\begin{array}{l}\text { Level of } \\
\text { Evidence }\end{array}$ & Surgical Procedure & Intervention & Treatment Groups & Dosage & Patients $(n)$ & Age (years) & Post-Op Pain Differences & $\begin{array}{l}\text { Post-Op Opioid } \\
\text { Consumption } \\
\text { Differences }\end{array}$ \\
\hline \multirow[t]{2}{*}{ Lierz et al., $2012^{63}$} & \multirow[t]{2}{*}{1} & \multirow[t]{2}{*}{$\begin{array}{c}\text { Therapeutic knee } \\
\text { arthroscopy }\end{array}$} & \multirow[t]{2}{*}{ Oral medication } & Etoricoxib & $\begin{array}{l}\text { One tablet of } 120 \mathrm{mg} \\
\text { etoricoxib, } 1 \text { hour before } \\
\text { anesthesia induction }\end{array}$ & 33 & $54 \pm 10$ & $\begin{array}{l}\text { VAS: Etoricoxib }< \\
\text { Placebo, at } 0 \text { hour and } \\
\text { from } 4 \text { to } 24 \text { hours. }\end{array}$ & $\begin{array}{c}\text { Total morphine } \\
\text { consumption: Etoricoxib } \\
\text { < Placebo, from } 2 \text { to } 24\end{array}$ \\
\hline & & & & Placebo & $\begin{array}{l}\text { One look-alike placebo } \\
\text { tablet, } 1 \text { hour before } \\
\text { anesthesia induction }\end{array}$ & 33 & $56 \pm 14$ & $\begin{array}{c}\text { Etoricoxib }=\text { Placebo, at } \\
2 \text { hours }\end{array}$ & hours \\
\hline \multirow[t]{2}{*}{$\begin{array}{l}\text { Mardani-Kivi et al., } \\
2013^{68}\end{array}$} & \multirow[t]{2}{*}{1} & \multirow{2}{*}{$\begin{array}{l}52 \% \text { isolated ACL } \\
\text { reconstruction (ACLR), } \\
48 \% \text { isolated partial } \\
\text { meniscectomy }\end{array}$} & \multirow[t]{2}{*}{ Oral medication } & Celecoxib & $\begin{array}{l}400 \text { mg celecoxib, } 2 \text { hours } \\
\text { prior to operation }\end{array}$ & 57 & $\begin{array}{l}\text { ACL: } 25.8 \pm 7.7 \\
\text { Meniscectomy: } \\
\quad 32.7 \pm 8\end{array}$ & \multirow{2}{*}{$\begin{array}{l}\text { VAS: Celecoxib }< \\
\text { Placebo, from } 6 \text { to } 24 \\
\text { hours, for both ACLR } \\
\text { and meniscectomy }\end{array}$} & $\begin{array}{l}\text { Opioid (Pethidine) } \\
\text { Consumption: Celecoxib } \\
\text { < Placebo, from 6-24 }\end{array}$ \\
\hline & & & & Placebo & $\begin{array}{l}\text { Identical placebo, } 2 \text { hours } \\
\text { prior to operation }\end{array}$ & 60 & $\begin{array}{c}\text { ACL: } 26.7 \pm 4.9 \\
\text { Meniscectomy: } \\
32.2 \pm 9.8\end{array}$ & & $\begin{array}{l}\text { hours, for meniscectomy } \\
\text { only. *Celecoxib }< \\
\text { Placebo, at } 6 \text { hours, for } \\
\text { ACLR. Celecoxib }< \\
\text { Placebo, at } 24 \text { hours }\end{array}$ \\
\hline \multirow[t]{4}{*}{$\begin{array}{l}\text { Tompkins et al., } \\
2011^{85}\end{array}$} & \multirow[t]{4}{*}{2} & \multirow[t]{4}{*}{ ACL reconstruction } & \multirow[t]{4}{*}{ Oral medication } & $\begin{array}{l}\text { Postoperative Zolpidem } \\
\text { (sleep-aid) with ibuprofen }\end{array}$ & $\begin{array}{c}7 \text { zolpidem tartrate tablets } \\
\text { (10 mg), taken once a day for } \\
7 \text { days after surgery. Also } 800 \\
\text { mg ibuprofen, taken every } 8 \\
\text { hours as needed. }\end{array}$ & 6 & 36.9 & \multirow[t]{4}{*}{$\begin{array}{l}\text { VAS: All groups are } \\
\text { equal, days } 0-7\end{array}$} & $\begin{array}{l}\text { Number of Vicodin } \\
\text { tablets: Zolpidem groups } \\
\text { < Placebo groups, days } \\
0-7 \text {. Ibuprofen did not } \\
\text { affect opioid }\end{array}$ \\
\hline & & & & $\begin{array}{l}\text { Postoperative Zolpidem } \\
\text { without ibuprofen }\end{array}$ & $\begin{array}{l}7 \text { zolpidem tartrate tablets } \\
(10 \mathrm{mg}) \text {, taken once a day for } \\
7 \text { days after surgery. }\end{array}$ & 7 & & & consumption. \\
\hline & & & & $\begin{array}{c}\text { Postoperative Placebo with } \\
\text { ibuprofen }\end{array}$ & $\begin{array}{c}7 \text { gelatin pills, taken once a } \\
\text { day for } 7 \text { days after surgery. } \\
\text { Also } 800 \mathrm{mg} \text { ibuprofen, } \\
\text { taken every } 8 \text { hours, as } \\
\text { needed. }\end{array}$ & 7 & 35.6 & & \\
\hline & & & & $\begin{array}{l}\text { Postoperative Placebo } \\
\text { without ibuprofen }\end{array}$ & $\begin{array}{l}7 \text { gelatin pills, taken once a } \\
\text { day for } 7 \text { days after surgery. }\end{array}$ & 9 & & & \\
\hline \multirow[t]{2}{*}{ Reda et al., $2016^{78}$} & \multirow[t]{2}{*}{1} & \multirow{2}{*}{$\begin{array}{l}\text { ACL reconstruction with } \\
\text { anatomical single-bundle } \\
\text { technique }\end{array}$} & \multirow[t]{2}{*}{$\begin{array}{l}\text { Nonpharmacological } \\
\text { intervention }\end{array}$} & A: Tourniquet & $\begin{array}{l}\text { Inflated to } 350 \mathrm{~mm} \text { mercury } \\
\qquad(64 \pm 8.7 \mathrm{~min})\end{array}$ & 29 & $25.5 \pm 4.0$ & \multirow{2}{*}{$\begin{array}{l}\text { VAS: *No Tourniquet }< \\
\text { Tourniquet, from } 4 \text { to } 10 \\
\text { hours. Tourniquet }=\text { No } \\
\text { Tourniquet, from } 16 \text { to } \\
22 \text { hours }\end{array}$} & \multirow{2}{*}{$\begin{array}{l}\text { Morphine Equivalent } \\
\text { Consumption: *No } \\
\text { Tourniquet }< \\
\text { Tourniquet, until } \\
\text { discharge }\end{array}$} \\
\hline & & & & B: No tourniquet & $\begin{array}{c}\text { Tourniquet not inflated; } \\
\text { received intra-articular } \\
\text { injection of } 60 \mathrm{cc}(250 \mathrm{cc} \\
\text { saline }+10 \mathrm{mg} \text { morphine }+1 \\
\text { mg Adrenaline) }\end{array}$ & 29 & $25.0 \pm 4.6$ & & \\
\hline \multirow[t]{2}{*}{$\begin{array}{l}\text { Hartwell et al., } \\
\quad 2020^{94}\end{array}$} & \multirow[t]{2}{*}{1} & \multirow[t]{2}{*}{$\begin{array}{l}\text { Knee arthroscopy with } \\
\text { partial meniscal } \\
\text { debridement }\end{array}$} & \multirow[t]{2}{*}{ Nonpharmacological } & $\begin{array}{l}\text { Electronic prescription: } \\
\text { automatically provided } \\
\text { opioids with multimodal pain } \\
\text { medications from pharmacist }\end{array}$ & $\begin{array}{l}20 \text { tablets of } 5 \text {-mg } \\
\text { oxycodone, automatically } \\
\text { provided after surgery }\end{array}$ & 48 & $45.0 \pm 12.3$ & \multirow{2}{*}{$\begin{array}{c}\text { VAS: Electronic } \\
\text { prescription }=\text { paper } \\
\text { prescription, at } 2,24 \text {, and } \\
48 \text { hours, as well as at } 7 \\
\text { and } 21 \text { days }\end{array}$} & \multirow[t]{2}{*}{$\begin{array}{l}\text { Number of Pills Taken: } \\
\text { Electronic prescription }= \\
\text { paper subscription, total } \\
\text { number of pills taken }\end{array}$} \\
\hline & & & & $\begin{array}{l}\text { Paper prescription: optional } \\
\text { opioids, only if absolutely } \\
\text { necessary for pain control }\end{array}$ & $\begin{array}{l}20 \text { tablets of } 5-\mathrm{mg} \\
\text { oxycodone, available if } \\
\text { needed after surgery }\end{array}$ & 47 & $43.6 \pm 12.8$ & & \\
\hline
\end{tabular}
scale. 
Table 4. Treatment Provided, Patient Population, Postoperative Pain, and Postoperative Opioid Consumption Summarized from All Included RCTs Regarding Hip Arthroscopy

\begin{tabular}{|c|c|c|c|c|c|c|c|c|c|}
\hline $\begin{array}{l}\text { Author, } \\
\text { Publication } \\
\text { Year }\end{array}$ & $\begin{array}{l}\text { Level of } \\
\text { Evidence }\end{array}$ & Surgical Procedure & Intervention & Treatment Groups & Dosage & Patients $(n)$ & Age (years) & $\begin{array}{l}\text { Post-Op Pain } \\
\text { Differences }\end{array}$ & $\begin{array}{l}\text { Post-Op Opioid } \\
\text { Consumption } \\
\text { Differences }\end{array}$ \\
\hline $\begin{array}{l}\text { Behrends } \\
\text { et al., } \\
2018^{34}\end{array}$ & 1 & $\begin{array}{c}\text { Femoroacetabular } \\
\text { impingement }\end{array}$ & Nerve block & $\begin{array}{c}\text { Fascia iliaca block } \\
\text { (FI) } \\
\text { Saline }\end{array}$ & $\begin{array}{c}40 \mathrm{~mL} .2 \% \text { ropivacaine } \\
\text { Saline }\end{array}$ & 38 & $\begin{array}{l}35 \pm 11 \\
32 \pm 9\end{array}$ & $\begin{array}{l}\text { NRS: FI=Saline, } \\
\text { from } 0 \text { to } 24 \text { hours }\end{array}$ & $\begin{array}{c}\text { Morphine and } \\
\text { Morphine } \\
\text { Equivalent } \\
\text { Consumption: FI = } \\
\text { Saline, from } 1 \text { to } 24 \\
\text { hours }\end{array}$ \\
\hline $\begin{array}{l}\text { Xing et al., } \\
2015^{88}\end{array}$ & 1 & $\begin{array}{l}\text { Femoroacetabular } \\
\text { impingement }\end{array}$ & Nerve block & $\begin{array}{l}\text { Femoral nerve block } \\
\text { (FNB) } \\
\text { Saline }\end{array}$ & $\begin{array}{c}20 \mathrm{~mL} .5 \% \text { bupivacaine } \\
\text { Saline }\end{array}$ & $\begin{array}{l}27 \\
23\end{array}$ & $\begin{array}{l}32 \pm 11 \\
31 \pm 8\end{array}$ & $\begin{array}{c}\text { VAS: FNB < } \\
\text { Saline, at } 6 \text { hours. } \\
{ }^{*} \text { FNB }<\text { Saline, from } \\
30 \text { min to } 1 \text { hour } \\
\text { and from } 2 \text { to } 4 \\
\text { hours. FNB }< \\
\text { Saline, at } 48 \text { hours } \\
\text { and day } 7 \text {. FNB }= \\
\text { Saline, at } 90 \\
\text { minutes and } 24 \\
\text { hours }\end{array}$ & $\begin{array}{c}\text { Total morphine } \\
\text { consumed: *FNB }< \\
\text { Saline, from } 24 \text { to } \\
48 \text { hours. FNB }= \\
\text { Saline, from } 1-24 \\
\text { hours and from days } \\
2-7\end{array}$ \\
\hline $\begin{array}{l}\text { Purcell et al., } \\
2019^{77}\end{array}$ & 1 & $\begin{array}{c}96 \% \text { labral repair, } 93 \% \\
\text { pincer resection, } 87 \% \\
\text { cam osteoplasty, } 83 \% \\
\text { capsular repair }\end{array}$ & Nerve block & $\begin{array}{c}\text { PB: Plain } \\
\text { bupivacaine } \\
\text { LB + PB: Liposomal } \\
\text { bupivacaine }+ \text { plain } \\
\text { bupivacaine }\end{array}$ & $\begin{array}{c}40 \mathrm{~mL} \text { of } .25 \% \text { plain } \\
\text { bupivacaine }(100 \mathrm{mg}) \\
20 \mathrm{~mL} \text { of } .5 \% \text { plain } \\
\text { bupivacaine }(100 \mathrm{mg})+ \\
20 \mathrm{~mL} \text { of liposomal } \\
\text { bupivacaine }(266 \mathrm{mg})\end{array}$ & $\begin{array}{l}37 \text { military } \\
\text { veterans } \\
33 \text { military } \\
\text { veterans }\end{array}$ & $\begin{array}{l}30.2 \\
32.8\end{array}$ & $\begin{array}{l}\text { DVPRS: } \mathrm{PB}=\mathrm{LB}+ \\
\mathrm{PB} \text {, from the PACU } \\
\text { until } 2 \text { weeks }\end{array}$ & $\begin{array}{c}\text { Oxycodone } \\
\text { Consumed: } \mathrm{PB}= \\
\mathrm{LB}+\mathrm{PB}, \text { from days } \\
1-14\end{array}$ \\
\hline $\begin{array}{l}\text { Glomset } \\
\text { et al., } \\
2020^{45}\end{array}$ & 1 & $\begin{array}{l}\text { Labral repair with both } \\
\text { acetabuloplasty and } \\
\text { femoroplasty }(76 \%) \text {, } \\
\text { labral repair with } \\
\text { acetabuloplasty or } \\
\text { femoroplasty }(14 \%) \text {, } \\
\text { other }(10 \%)\end{array}$ & $\begin{array}{l}\text { Nerve block vs. } \\
\text { intra-articular } \\
\text { injection }\end{array}$ & $\begin{array}{l}\text { Intra-articular (IA) } \\
\text { injection, at } \\
\text { completion of } \\
\text { surgery }\end{array}$ & $\begin{array}{l}\text { Up to } 60 \mathrm{~mL} .35 \% \\
\text { ropivacaine at } 3 \mathrm{mg} / \mathrm{kg} \text {, } \\
\text { with } 100-\mathrm{mg} \text { clonidine } \\
\text { (per } 60 \mathrm{~mL} \text { ) and } \\
\text { epinephrine } 1: 400,000 \\
20 \mathrm{~mL} .5 \% \text { ropivacaine, } \\
\text { at the end of surgery }\end{array}$ & 43 & $36.8 \pm 12.1$ & $\begin{array}{c}\text { VAS: } \mathrm{FIB}=\mathrm{IA} \text {, in } \\
\text { PACU, and at } 2 \\
\text { weeks, } 6 \text { weeks, and } \\
3 \text { months }\end{array}$ & $\begin{array}{c}\text { Morphine } \\
\text { Equivalents: FIB }= \\
\text { IA, in PACU }\end{array}$ \\
\hline $\begin{array}{l}\text { Cogan et al., } \\
2020^{40}\end{array}$ & 1 & $\begin{array}{l}\text { Hip arthroscopy, labral } \\
\text { repair, and } \\
\text { acetabuloplasty }\end{array}$ & $\begin{array}{l}\text { Intra-articular } \\
\text { injection }\end{array}$ & $\begin{array}{l}\text { M + C: Morphine }+ \\
\text { clonidine } \\
\text { Control: Normal } \\
\text { saline }\end{array}$ & $\begin{array}{c}11 \mathrm{~mL} \text { of } 10 \mathrm{mg} \\
\text { morphine, and } 100 \mathrm{mcg} \\
\text { clonidine, in .9\% NaCl } \\
\text { solution, at the } \\
\text { conclusion of arthroscopy } \\
11 \mathrm{~mL} \text { of } .9 \% \mathrm{NaCl} \\
\text { solution, at conclusion of } \\
\text { arthroscopy }\end{array}$ & 33 & 36 & $\begin{array}{c}\text { VAS: } \mathrm{M}+\mathrm{C}= \\
\text { control, in PACU, } \\
\text { and at } 7,18,24 \text {, and } \\
48 \text { hours, as well as } \\
\text { at } 7 \text { days }\end{array}$ & $\begin{array}{c}\text { Morphine } \\
\text { Equivalents: } M+ \\
C=\text { control, in } \\
\text { PACU, and at } 7,18 \text {, } \\
\text { 24, and } 48 \text { hours, as } \\
\text { well as at } 7 \text { days }\end{array}$ \\
\hline
\end{tabular}




\begin{tabular}{|c|c|c|c|c|c|c|c|c|c|}
\hline $\begin{array}{l}\text { Author, } \\
\text { Publication } \\
\text { Year }\end{array}$ & $\begin{array}{l}\text { Level of } \\
\text { Evidence }\end{array}$ & Surgical Procedure & Intervention & Treatment Groups & Dosage & Patients $(n)$ & Age (years) & $\begin{array}{l}\text { Post-Op Pain } \\
\text { Differences }\end{array}$ & $\begin{array}{l}\text { Post-Op Opioid } \\
\text { Consumption } \\
\text { Differences }\end{array}$ \\
\hline $\begin{array}{l}\text { Shlaifer et al., } \\
2017^{82}\end{array}$ & 1 & $\begin{array}{l}\text { Femoroacetabular } \\
\text { impingement }\end{array}$ & $\begin{array}{l}\text { Surgical Site } \\
\text { Injection }\end{array}$ & $\begin{array}{c}\text { Periacetabular } \\
\text { Injection } \\
\text { Intra-articular Hip } \\
\text { Injection }\end{array}$ & $\begin{array}{c}20 \mathrm{~mL} .5 \% \text { bupivacaine } \\
\text { with epi }(1: 200,000) \text {, } \\
\text { before surgery } \\
20 \mathrm{~mL} .5 \% \text { bupivacaine } \\
\text { with epi ( } 1: 200,000) \\
\text { before surgery }\end{array}$ & 21 & $39.6 \pm 16.1$ & $\begin{array}{c}\text { VAS: Periacetabular } \\
<\text { Intra-articular, } 30 \\
\text { min. *Periacetabular } \\
<\text { Intra-articular, at } \\
18 \text { hours } \\
\text { Periacetabular = } \\
\text { Intra-articular, from } \\
1 \text { to } 12 \text { hours, and } \\
\text { from days } 1 \text { to } 14\end{array}$ & $\begin{array}{c}\text { Morphine } \\
\text { Equivalents: } \\
\text { Periacetabular }= \\
\text { Intra-articular, until } \\
\text { discharge and days } \\
1-7\end{array}$ \\
\hline $\begin{array}{l}\text { Kahlenberg } \\
\text { et al., } \\
2017^{51}\end{array}$ & 1 & $\begin{array}{c}61 \% \text { labral repair, } 24 \% \\
\text { labral repair with } \\
\text { acetabular osteoplasty, } \\
11 \% \text { other }\end{array}$ & Oral medication & $\begin{array}{l}\text { Celecoxib } \\
\text { Placebo }\end{array}$ & $\begin{array}{l}1 \text { hour pre-op: } 2 \text { pills, } 200 \\
\text { mg celecoxib each } \\
1 \text { hour pre-op: } 2 \text { lactose- } \\
\text { based placebo pills }\end{array}$ & $\begin{array}{l}50 \\
48\end{array}$ & $\begin{array}{l}34.2 \\
35.8\end{array}$ & $\begin{array}{l}\text { VAS: Celecoxib }< \\
\text { control at } 1 \text { hour, } \\
\text { and Celecoxib } \\
\text { almost }<\text { control at } \\
2 \text { hours }(P=.06) \text {. } \\
\text { Celecoxib }=\text { control, } \\
\text { until discharge }\end{array}$ & $\begin{array}{c}\text { Morphine } \\
\text { Equivalents: } \\
\text { Celecoxib }=\text { control, } \\
\text { in PACU }\end{array}$ \\
\hline $\begin{array}{l}\text { Zhang et al., } \\
2014^{90}\end{array}$ & 1 & $\begin{array}{c}\text { Femoroacetabular } \\
\text { impingement with } \\
\text { labral tears }\end{array}$ & Oral medication & $\begin{array}{l}\text { Celecoxib } \\
\text { Placebo }\end{array}$ & $\begin{array}{l}200 \text { mg celecoxib } 1 \text { hour } \\
\text { before surgery } \\
200 \text { mg placebo } 1 \text { hour } \\
\text { before surgery }\end{array}$ & $\begin{array}{l}27 \\
26\end{array}$ & $\begin{array}{l}41.0 \pm 4.9 \\
43.5 \pm 5.1\end{array}$ & $\begin{array}{l}\text { VAS: Celecoxib }= \\
\text { Placebo, in recovery } \\
\text { room. Celecoxib }< \\
\text { Placebo, from } 12 \text { to } \\
24 \text { hours }\end{array}$ & $\begin{array}{c}\text { Number of Narcotic } \\
\text { Pills Used: Celecoxib } \\
<\text { Placebo, in } \\
\text { recovery room }\end{array}$ \\
\hline
\end{tabular}

Findings with a $P$ value $<.01$ are marked with an asterisk $\left(^{*}\right)$, and findings with a $P$ value $<.001$ are in bold.

DVPRS, defense and veterans pain rating scale; VAS, visual analog scale; NRS, numeric pain rating scale, PACU, postanesthesia care unit. 


\section{Postoperative Interventions for Pain Management}

Ten studies evaluated interventions for postoperative pain management protocols, six in shoulder arthroscopy, ${ }^{61,66,71,75,83}$ four in knee arthroscopy, ${ }^{29,85,91,94}$ and none in hip arthroscopy. Notable in shoulder arthroscopy, Yun et al. ${ }^{89}$ found that subacromial patientcontrolled analgesia (PCA) provided postoperatively for 48 hours to rotator cuff repair patients provided better pain relief at 1 hour postoperatively compared to IV PCA, while opioid consumption was similar. Merivirta et ll. $^{71}$ and Oh et al. $^{75}$ found that a 72 -hour postoperative fentanyl batch provided similar outcomes to a bupivacaine infusion and that Nefopam IV PCA provided similar outcomes to a Ketorolac IV PCA, respectively. For knee arthroscopy, Tompkins et al. ${ }^{85}$ found that providing the sleep aid Zolpidem (10 mg) to ACL reconstruction patients for days 0-7 postoperatively decreased opioid consumption, while not affecting pain relief. Amin et al. ${ }^{29}$ compared three groups of intraarticular PCA for ACL reconstruction patients: an RM group $(.25 \%$ ropivacaine $+.2 \mathrm{mg} / \mathrm{mL}$ morphine), an RMX group (ropivacaine + morphine $+1 \mathrm{mg} / \mathrm{mL}$ xefocam [i.e. Lornoxicam]), and a control group. They found that the RMX group had the best pain relief from 8 to 24 hours postoperatively and dramatically less opioid consumption than the RM group at 24 hours.

\section{Nonpharmacological Interventions}

Six studies evaluated nonpharmacological interventions for pain management, four for shoulder arthroscopy, ${ }^{39,61,66,84}$ two for knee arthroscopy, ${ }^{78,94}$ and none for hip arthroscopy. Both Cho et al. ${ }^{39}$ (orally and written) and Syed et al. ${ }^{84}$ (2-minute video) provided preoperative opioid education to rotator cuff repair patients, and both studies found opioid education to decrease postoperative pain and opioid consumption throughout short-term $(<1$ week) and mid-term (up to 3 months) recovery. Mahure et al. ${ }^{66}$ also evaluated rotator cuff repair patients, finding that using a transcutaneous electrical nerve stimulation (TENS) unit throughout the first postoperative week can decrease pain and opioid consumption throughout that week. However, Kraeutler et al. ${ }^{61}$ found no difference in outcomes between shoulder arthroscopy patients who used compressive cryotherapy versus a standard ice wrap postoperatively. Reda et al. ${ }^{78}$ showed that for ACL reconstruction patients, the use of a tourniquet negatively affects outcomes, with tourniquet use showing increased pain from 4 to 10 hours postoperatively and increased opioid consumption until discharge. Also, Hartwell et al. ${ }^{94}$ evaluated whether the mode of prescription affects postoperative outcomes by providing one group of patients with optional paper prescriptions for 20 tablets of $5 \mathrm{mg}$ oxycodone and the other group with the same prescriptions automatically provided (not optional) from the pharmacist. However, there were no differences in pain or opioid consumption at any time point up to 21 days postoperatively.

\section{Discussion}

On the basis of current evidence, we recommend interscalene nerve blocks with a dexamethasonedexmedetomidine combination for rotator cuff repair, a proximal continuous adductor canal block for ACL reconstruction, and local infiltration analgesia (e.g., periacetabular injection with $20 \mathrm{~mL}$ of $.5 \%$ bupivacaine) for hip arthroscopy. Several oral medications appear to be optimal as well, such as $150 \mathrm{mg}$ Pregabalin for shoulder arthroscopy, $400 \mathrm{mg}$ Celecoxib for knee arthroscopy, and $200 \mathrm{mg}$ Celecoxib for hip arthroscopy. There is promising evidence for the use of various nonpharmacological modalities, specifically preoperative opioid education for rotator cuff repair patients; however more clinical trials evaluating nonpharmacological interventions should be performed.

\section{Shoulder Arthroscopy}

A number of different nerve block locations and formulations were examined following shoulder arthroscopy. While bupivacaine alone was shown to reduce postoperative opioid consumption, the addition of dexamethasone to the interscalene block resulted in even lower postoperative pain for rotator cuff repair patients. ${ }^{32}$ However, Kang et al. ${ }^{54}$ compared IV dexamethasone to IV dexamethasone-dexmedetomidine and showed that a combination of dexamethasone and dexmedetomidine (IV dexamethasone .11 mg/kg + IV dexmedetomidine $1.0 \mu \mathrm{g} / \mathrm{kg}$ ) decreased postoperative pain and opioid consumption in a cohort of $73 \%$ rotator cuff repair patients. These findings were further supported by Bengisun et al., ${ }^{35}$ who reported similarly superior outcomes with the addition of dexmedetomidine to an interscalene levobupivacaine and epinephrine block; however, this study involved a cohort of subacromial decompression patients. While other studies conflicted on the effects of block location, the overall trend was that interscalene brachial plexus blocks performed equal to or greater than supraclavicular and suprascapular blocks for both pain and opioid control. ${ }^{26,31,74}$ Cabaton et al. ${ }^{37}$ had results opposing the superiority of the interscalene brachial plexus block for rotator cuff repair patients, as they reported less opioid consumption after injection at a supraclavicular site; however, these authors used a levobupivacaine and clonidine block rather than a ropivacaine formulation.

Nerve blocks appear to be the optimal pain management modality for shoulder arthroscopy, as the bupivacaine interscalene brachial plexus block was superior to bupivacaine intra-articular injections for both pain control and opioid consumption in a cohort of mostly rotator cuff repair $(67 \%)$ and Bankart repair $(20 \%)$ 
patients. ${ }^{28}$ However, intra-articular injections for shoulder arthroscopy may be optimized by using morphine (20 mg morphine/10 mL) or magnesium (1 g magnesium sulfate in $10 \mathrm{~mL}$ saline). ${ }^{57,80}$ Before a clear clinical recommendation can be provided, further highquality studies comparing morphine, magnesium, bupivacaine, and any other viable intra-articular injections formulations should be conducted. Lastly, preemptively providing the oral medication Pregabalin can help patients after shoulder arthroscopy. MardaniKivi et al. ${ }^{67}$ found that $600 \mathrm{mg}$ Gabapentin significantly decreased postoperative opioid consumption in Bankart repair patients; however, Spence et al. ${ }^{83}$ had conflicting results in a military patient population undergoing unspecified shoulder arthroscopy. Meanwhile, Ahn et al. ${ }^{27}$ showed that $150 \mathrm{mg}$ Pregabalin decreased both postoperative pain and opioid consumption compared to placebo in a cohort of $75 \%$ rotator cuff repair patients, ultimately providing pain relief, while Gabapentin did not significantly decrease pain in either aforementioned study.

\section{Knee Arthroscopy}

Postoperative pain following knee arthroscopy was reported as equivalent, and opioid consumption appears similar, when comparing ropivacaine as an adductor canal block (ACB) to a femoral nerve block (FNB) in ACL reconstruction patients. ${ }^{24,33,65}$ This is supported by a network meta-analysis performed by Davey et al. ${ }^{95}$ who found that nerve blocks are efficacious for ACL reconstruction, but that no specific nerve block proved superior. However, proximal ACBs were found to significantly reduce pain within the first 6 hours after ACL reconstruction compared to middle and distal ACBs. ${ }^{25}$ In addition, continuous nerve blocks were shown to reduce pain between hours 4 and 12 after ACL reconstruction compared to intermittent ACBs. ${ }^{92}$ Proximal and continuous ACBs were also superior to comparison groups for the minimization of postoperative opioid consumption, suggesting that the proximal and continuous ACB may be the most effective modality for pain management for ACL reconstruction. ${ }^{25,92}$ Regarding intra-articular injections, Davey et al. ${ }^{95}$ also found that intra-articular injections with bupivacaine decrease pain for up to 12 hours postoperatively, while also decreasing postoperative opioid consumption. Unfortunately, the optimal pain management for meniscectomy is less clear, as several injections provided better outcomes than placebo, but no studies have compared these interventions to determine superiority. ${ }^{60,79,93}$ Providing either $120 \mathrm{mg}$ Etoricoxib and $400 \mathrm{mg}$ Celecoxib preoperatively leads to significant improvements in both pain and opioid consumption for various knee arthroscopy procedures. ${ }^{63,68,91}$ An RCT comparing preoperative Etoricoxib and Celecoxib would help clarify whether one oral medication is superior to the other for knee arthroscopy.

\section{Hip Arthroscopy}

Following hip arthroscopy, Xing et al. ${ }^{88}$ found a decrease in postoperative pain relief and opioid consumption with the use of a bupivacaine femoral nerve block compared to saline controls. However, the nerve block was also associated with a significant increase in the risk of falls within the first 24 hours following the procedure, which ultimately led to the discontinuation of bupivacaine femoral nerve blocks for outpatient hip arthroscopy procedures at the respective institution. ${ }^{88}$ The fascia iliaca block also may be an inferior form of pain management, as the fascia iliaca block provides equal pain relief compared to saline placebo. ${ }^{34,45,77}$ No RCTs included in this study evaluated the lumbar plexus nerve block; however, it is possible that the lumbar plexus nerve block may provide the best clinical outcomes of the common hip arthroscopy nerve blocks. ${ }^{96,97}$ For example, YaDeau et al. ${ }^{97}$ described in a brief report of an RCT that the addition of a lumbar plexus nerve block with a combined spinal epidural leads to significantly decreased postoperative pain compared to only receiving the spinal epidural. Also, in a retrospective cohort study, Wolff et al. ${ }^{96}$ found that a lumbar plexus nerve block with general anesthesia leads to much less postoperative pain than a fascia iliaca nerve block with general anesthesia. However, until several high-quality RCTs compare the lumbar plexus nerve block to other viable nerve blocks, the lumbar plexus nerve block should not be considered the gold standard of nerve blocks for hip arthroscopy.

Interestingly, the network meta-analyses performed by Kunze et al. ${ }^{15}$ suggest that local infiltration anesthesia is more effective than nerve block for limiting both postoperative pain and opioid consumption for hip arthroscopy. Specifically regarding local infiltration anesthesia within our included studies, Shlaifer et al. ${ }^{82}$ reported a decrease in postoperative pain if $20 \mathrm{~mL}$ of $.5 \%$ bupivacaine was used as periacetabular injection as opposed to an intra-articular injection. Interestingly, the use of morphine and clonidine as an intra-articular injection provided equivalent pain relief and opioid consumption compared to placebo. ${ }^{40}$ Lastly in regard to oral medication, while the use of oral celecoxib resulted in decreased postoperative pain compared to controls, only Zhang et al. ${ }^{90}$ reported a decrease in postoperative opioid consumption. ${ }^{51,90}$

\section{Nonpharmacological Interventions}

Several nonpharmacological interventions appear to provide clinical benefit for arthroscopic surgery patients. Preoperative patient opioid education of any form appears to provide clear benefits regarding both postoperative pain and opioid consumption for rotator cuff 
repair patients. ${ }^{39,84}$ Syed et al. ${ }^{84}$ also found that preoperative education patients were more than 2 times more likely to stop their narcotic use by 3 months postoperatively. Considering how easily preoperative opioid education was provided by Syed et al., ${ }^{84}$ providing patients with an educational 2-minute video and a handout, preoperative opioid education can be a realistic and beneficial intervention for managing postoperative pain and opioid consumption. The use of a TENS unit throughout the first postoperative week may also help rotator cuff repair patients; however, the feasibility depends on the finances of each institution and adherence of the patient. ${ }^{66}$ For ACL reconstruction, abandoning the tourniquet appears advantageous. ${ }^{78}$ Surgeons that feel comfortable abandoning tourniquet use may be able to decrease postoperative pain and opioid consumption for ACL reconstruction patients. ${ }^{78}$ Unfortunately, several nonpharmacological interventions do not appear to provide additional pain relief or minimize opioid consumption, such as cryotherapy for shoulder arthroscopy patients or optional paper prescriptions for knee arthroscopy patients. ${ }^{61,94}$

\section{Postoperative Interventions for Pain Management}

Similarly, several postoperative interventions may prove beneficial for certain arthroscopic surgery populations. For example, rotator cuff patients have decreased postoperative pain when given subacromial PCA instead of IV PCA for 48 hours postoperatively. ${ }^{89}$ Similarly, postoperative pain and opioid consumption can be decreased for ACL reconstruction patients by including ropivacaine, morphine, and xefocam (Lornoxicam) for intra-articular PCA. ${ }^{29}$ ACL reconstruction patients may also benefit from the use of a sleep aid such as Zolpidem $10 \mathrm{mg} .{ }^{85}$ While taking Zolpidem postoperatively days 0-7 did not decrease pain, opioid consumption significantly decreased. ${ }^{85}$

\section{Clinical Recommendations}

Development of an optimal analgesic strategy based on the articles examined is difficult due to the paucity of direct comparisons between various treatment modalities. However, each anatomic location demonstrated similar trends in regard to maximizing pain relief and minimizing opioid consumption. First, a distinct nerve block may be superior at each surgical location. Interscalene brachial plexus blocks with ropivacaine appear superior for rotator cuff repair, ${ }^{17,32,37,87,44}$ while proximal continuous adductor canal blocks were superior for ACL reconstruction. ${ }^{24,25,33,65,92,95}$ Second, certain oral medications taken preoperatively may limit both postoperative pain and opioid consumption, with 150 mg Pregabalin being optimal for shoulder arthroscopy, ${ }^{27,67,83} 400 \mathrm{mg}$ Celecoxib for knee arthroscopy, ${ }^{63,68}$ and $200 \mathrm{mg}$ Celecoxib for hip arthroscopy. ${ }^{51,90}$ Lastly, several nonpharmacological interventions have the potential to improve pain management with minimal risk to the patient. For example, preoperative patient opioid education, minimization of tourniquet use, and postoperative TENS unit usage can decrease pain and opioid consumption, while replacing electronic prescriptions with paper prescriptions may minimize the amount of unused opioid tablets available to the public. ${ }^{39,66,84,94}$

\section{Limitations}

This review is not without limitations. The included studies were separated on the basis of anatomic location. However, many studies included a number of different surgical procedures at the anatomic site. In addition, studies used different drug formulations for pain control and different morphine equivalents for opioid consumption. As a result, directly comparing outcomes between studies was not feasible. Also, the heterogeneity of data prevented pooling of results, which weakens the strength of the study conclusions. Plus, complications and patient-reported outcomes were not evaluated in this systematic review. Finally, minimal clinically important differences (MCIDs) were not evaluated in this study, only statistical significance.

\section{Conclusions}

Many multimodal pain management protocols offer improved pain control and decreased opioid consumption after arthroscopic surgery. On the basis of the current evidence, we recommend an interscalene nerve block with a dexamethasone-dexmedetomidine combination for rotator cuff repair, a proximal continuous adductor canal block for ACL reconstruction, and local infiltration analgesia (e.g., periacetabular injection with $20 \mathrm{~mL}$ of $.5 \%$ bupivacaine) for hip arthroscopy. When evaluating oral medication: the evidence supports 150 mg Pregabalin for shoulder arthroscopy, $400 \mathrm{mg}$ Celecoxib for knee arthroscopy, and $200 \mathrm{mg}$ Celecoxib for hip arthroscopy, all taken preoperatively. There is promising evidence for the use of various nonpharmacological modalities, specifically preoperative opioid education for rotator cuff repair patients; however, more clinical trials evaluating nonpharmacological interventions should be performed.

\section{References}

1. Chisholm-Burns MA, Spivey CA, Sherwin E, Wheeler J, Hohmeier K. The opioid crisis: Origins, trends, policies, and the roles of pharmacists. Am J Health-Syst Pharm 2019;76:424-435.

2. Skolnick P. The opioid epidemic: Crisis and solutions. Annu Rev Pharmacol Toxicol 2018;58:143-159.

3. Chen LH. Drug-poisoning deaths involving opioid analgesics: United States, 1999-2011. NCHS Data Brief 2014;(166):1-8. 
4. Dart RC, Surratt HL, Cicero TJ, et al. Trends in opioid analgesic abuse and mortality in the United States. $N$ Engl J Med 2015;372:241-248.

5. Vital Signs: Overdoses of Prescription Opioid Pain Relievers-United States, 1999-2008. MMWR Morb Mortal Wkly Rep 201 1;60:1487-1492.

6. Opioid data analysis and resources, https://www.cdc.gov/ opioids/data/analysis-resources.html. Accessed October 1, 2020.

7. Daubresse M, Chang H-Y, Yu Y, et al. Ambulatory diagnosis and treatment of nonmalignant pain in the United States, 2000-2010. Med Care 2013;51:870-878.

8. Gan TJ. Poorly controlled postoperative pain: prevalence, consequences, and prevention. J Pain Res 2017;10: 2287-2298.

9. Gan TJ, Habib AS, Miller TE, White W, Apfelbaum JL. Incidence, patient satisfaction, and perceptions of postsurgical pain: results from a US national survey. Curr Med Res Opin 2014;30:149-160.

10. Meissner W, Mescha S, Rothaug J, et al. Quality improvement in postoperative pain management. Dtsch Ärztebl Int 2008;105:865-870.

11. Calvo E, Torres MD, Morcillo D, Leal V. Rotator cuff repair is more painful than other arthroscopic shoulder procedures. Arch Orthop Trauma Surg 2019;139:669-674.

12. Volkow ND, McLellan TA, Cotto JH, Karithanom M, Weiss SRB. Characteristics of opioid prescriptions in 2009. JAMA 2011;305:1299-1301.

13. Practice guidelines for acute pain management in the perioperative setting: An updated report by the American Society of Anesthesiologists Task Force on Acute Pain Management. Anesthesiology 2012;116:248-273.

14. Secrist ES, Freedman KB, Ciccotti MG, Mazur DW, Hammoud S. Pain management after outpatient anterior cruciate ligament reconstruction: A systematic review of randomized controlled trials. Am J Sports Med 2016;44: 2435-2447.

15. Kunze KN, Polce EM, Lilly DT, et al. Adjunct analgesia reduces pain and opioid consumption after hip arthroscopy: A systematic review of randomized controlled trials. Am J Sports Med 2020;48:3638-3651.

16. Hurley ET, Maye AB, Thompson K, et al. Pain control after shoulder arthroscopy: A systematic review of randomized controlled trials with a network meta-analysis. Am J Sports Med 2021;49:2262-2271.

17. Warrender WJ, Syed UAM, Hammoud S, et al. Pain management after outpatient shoulder arthroscopy: A systematic review of randomized controlled trials. Am J Sports Med 2017;45:1676-1686.

18. Moher D, Shamseer L, Clarke M, et al. Preferred reporting items for systematic review and meta-analysis protocols (PRISMA-P) 2015 statement. Syst Rev 2015;4:1.

19. Abou-Setta AM, Beaupre LA, Rashiq S, et al. Comparative effectiveness of pain management interventions for hip fracture: A systematic review. Ann Intern Med 2011;155: 234-245.

20. Brubaker L, Kendall L, Reina E. Multimodal analgesia: A systematic review of local NSAIDs for non-ophthalmologic postoperative pain management. Int J Surg 2016;32:158-166.

21. Lee $Y, Y u J$, Doumouras $A G$, et al. Intravenous acetaminophen versus placebo in post-bariatric surgery multimodal pain management: A meta-analysis of randomized controlled trials. Obes Surg 2019;29:1420-1428.

22. Tong Y, Fernandez L, Bendo JA, Spivak JM. Enhanced recovery after surgery trends in adult spine surgery: A systematic review. Int J Spine Surg 2020;14:623-640.

23. Higgins JPT, Altman DG, Gøtzsche PC, et al. The Cochrane Collaboration's tool for assessing risk of bias in randomised trials. BMJ 201 1;343:d5928.

24. Abdallah FW, Whelan DB, Chan VW, et al. Adductor canal block provides noninferior analgesia and superior quadriceps strength compared with femoral nerve block in anterior cruciate ligament reconstruction. Anesthesiology 2016;124:1053-1064.

25. Abdallah FW, Mejia J, Prasad GA, et al. Opioid- and motor-sparing with proximal, mid-, and distal locations for adductor canal block in anterior cruciate ligament reconstruction: A randomized clinical trial. Anesthesiology 2019;131:619-629.

26. Abdallah FW, Wijeysundera DN, Laupacis A, et al. Subomohyoid anterior suprascapular block versus interscalene block for arthroscopic shoulder surgery: A multicenter randomized trial. Anesthesiology 2020;132: 839-853.

27. Ahn S, Byun SH, Park K, Ha JL, Kwon B, Kim JC. Analgesic efficacy of preemptive pregabalin administration in arthroscopic shoulder surgery: A randomized controlled trial. Can J Anaesth J Can Anesth 2016;63: 283-289.

28. Aksu R, Biçer C, Ülgey A, et al. Comparison of interscalene brachial plexus block and intra-articular local anesthetic administration on postoperative pain management in arthroscopic shoulder surgery. Braz J Anesthesiol 2015;65:222-229.

29. Amin S, Yosry M, El DI. The effect of the addition of lornoxicam (xefocam) intrarticularly on the WOMAC Scale in patients undergoing arthroscopic anterior cruciate ligament reconstruction. Middle East J Anaesthesiol 2011;21:15-21.

30. Arti H, Mehdinasab SA. The comparison effects of intraarticular injection of different opioids on postoperative pain relieve after arthroscopic anterior cruciate ligament reconstruction: A randomized clinical trial study. J Res Med Sci $2011 ; 16: 1176-1182$.

31. Auyong DB, Hanson NA, Joseph RS, Schmidt BE, Slee AE, Yuan SC. Comparison of anterior suprascapular, supraclavicular, and interscalene nerve block approaches for major outpatient arthroscopic shoulder surgery: A randomized, double-blind, noninferiority trial. Anesthesiology 2018;129:47-57.

32. Baessler AM, Moor M, Conrad DJ, Creighton J, Badman BL. Single-shot liposomal bupivacaine reduces postoperative narcotic use following outpatient rotator cuff repair: A prospective, double-blinded, randomized controlled trial. J Bone Joint Surg Am 2020;102:1985-1992.

33. Bailey L, Griffin J, Elliott M, et al. Adductor canal nerve versus femoral nerve blockade for pain control and quadriceps function following anterior cruciate ligament reconstruction with patellar tendon autograft: A prospective randomized trial. Arthroscopy 2019;35:921-929.

34. Behrends M, Yap EN, Zhang AL, et al. Preoperative fascia iliaca block does not improve analgesia after arthroscopic 
hip surgery, but causes quadriceps muscles weakness: A randomized, double-blind trial. Anesthesiology 2018;129: 536-543.

35. Bengisun ZK, Ekmekçi P, Akan B, Köroğlu A, Tüzüner F. The effect of adding dexmedetomidine to levobupivacaine for interscalene block for postoperative pain management after arthroscopic shoulder surgery. Clin J Pain 2014;30: 1057-1061.

36. Bjørnholdt KT, Mønsted PN, Søballe K, Nikolajsen L. Dexamethasone for pain after outpatient shoulder surgery: a randomised, double-blind, placebo-controlled trial. Acta Anaesthesiol Scand 2014;58:751-758.

37. Cabaton J, Nové-Josserand L, Mercadal L, Vaudelin T. Analgesic efficacy of ultrasound-guided interscalene block vs. supraclavicular block for ambulatory arthroscopic rotator cuff repair: A randomised noninferiority study. Eur J Anaesthesiol 2019;36:778-786.

38. Choromanski DW, Patel PS, Frederick JM, Lemos SE, Chidiac EJ. The effect of continuous interscalene brachial plexus block with $0.125 \%$ bupivacaine vs $0.2 \%$ ropivacaine on pain relief, diaphragmatic motility, and ventilatory function. J Clin Anesth 2015;27:619-626.

39. Cho C-H, Song K-S, Min B-W, et al. Multimodal approach to postoperative pain control in patients undergoing rotator cuff repair. Knee Surg Sports Traumatol Arthrosc $2011 ; 19: 1744-1748$.

40. Cogan CJ, Tjong VK, Dunne KF, Sahota S, Tuttle J, Terry MA. Intra-articular morphine and clonidine injection after hip arthroscopy: A randomized, triple-blind controlled trial. Ambul Surg 2020;26:5-9.

41. DeMarco JR, Componovo R, Barfield WR, Liles L, Nietert P. Efficacy of augmenting a subacromial continuous-infusion pump with a preoperative interscalene block in outpatient arthroscopic shoulder surgery: a prospective, randomized, blinded, and placebo-controlled study. Arthroscopy $2011 ; 27: 603-610$.

42. Espelund M, Fomsgaard JS, Haraszuk J, Dahl JB, Mathiesen $\mathrm{O}$. The efficacy of adductor canal blockade after minor arthroscopic knee surgery-a randomised controlled trial. Acta Anaesthesiol Scand 2014;58:273-280.

43. Espelund M, Grevstad U, Jaeger P, et al. Adductor canal blockade for moderate to severe pain after arthroscopic knee surgery: a randomized controlled trial. Acta Anaesthesiol Scand 2014;58:1220-1227.

44. Faria-Silva R, de Rezende DC, Ribeiro JM, et al. Association of clonidine and ropivacaine in brachial plexus block for shoulder arthroscopy. Braz J Anesthesiol Elsevier 2016;66:335-340.

45. Glomset JL, Kim EJ, Tokish JM, et al. Reduction of hip arthroscopy post-operative pain using ultrasound-guided fascia-iliaca block: A prospective randomized control trial. Orthop J Sports Med 2020;48:682-688.

46. Hanson NA, Derby RE, Auyong DB, et al. Ultrasoundguided adductor canal block for arthroscopic medial meniscectomy: a randomized, double-blind trial. Can J Anaesth 2013;60:874-880.

47. Hartwell MJ, Selley RS, Terry MA, Tjong VK. Can We Eliminate Opioid Medications for Postoperative Pain Control? A Prospective, Surgeon-Blinded, Randomized Controlled Trial in Knee Arthroscopic Surgery. Am J Sports Med 2020;48:2711-2717.
48. Hsu LP, Oh S, Nuber GW, et al. Nerve block of the infrapatellar branch of the saphenous nerve in knee arthroscopy: a prospective, double-blinded, randomized, placebo-controlled trial. J Bone Joint Surg Am 2013;95: 1465-1472.

49. Jeske H-C, Kralinger F, Wambacher M, et al. A randomized study of the effectiveness of suprascapular nerve block in patient satisfaction and outcome after arthroscopic subacromial decompression. Arthroscopy 201 1;27:1323-1328.

50. Kager I, Mousa SA, Sieper J, Stein C, Pipam W, Likar R. Blockade of intra-articular adrenergic receptors increases analgesic demands for pain relief after knee surgery. Rheumatol Int 2011;31:1299-1306.

51. Kahlenberg CA, Patel RM, Knesek M, Tjong VK, Sonn K, Terry MA. Efficacy of celecoxib for early postoperative pain management in hip arthroscopy: A prospective randomized placebo-controlled study. Arthroscopy 2017;33: $1180-1185$.

52. Kahn RL, Cheng J, Gadulov Y, Fields KG, YaDeau JT, Gulotta LV. Perineural low-dose dexamethasone Prolongs interscalene block analgesia with bupivacaine compared with systemic dexamethasone: A randomized trial. Reg Anesth Pain Med 2018;43:572-579.

53. Kang R, Jeong JS, Yoo JC, et al. Effective dose of intravenous dexmedetomidine to prolong the analgesic duration of interscalene brachial plexus block: A single-center, prospective, double-blind, randomized controlled trial. Reg Anesth Pain Med 2018;43:488-495.

54. Kang RA, Jeong JS, Yoo JC, et al. Improvement in postoperative pain control by combined use of intravenous dexamethasone with intravenous dexmedetomidine after interscalene brachial plexus block for arthroscopic shoulder surgery: A randomised controlled trial. Eur J Anaesthesiol 2019;36:360-368.

55. Kataria S, Mitra S, Saroa R, Jindal S, Gupta R. A randomized double blinded trial comparing dexmedetomidine with dexamethasone as an adjunct to ropivacaine in ultrasound guided interscalene block for arthroscopic shoulder surgery. Asian J Anesthesiol 2019;57:10-18.

56. Keller RA, Birns ME, Cady AC, Limpisvasti O, Banffy MB. Posterior capsule injection of local anesthetic for postoperative pain control after ACL reconstruction: a prospective, randomized trial. Knee Surg Sports Traumatol Arthrosc 2019;27:822-826.

57. Khashan M, Dolkart O, Amar E, et al. Effect of preemptive intra-articular morphine and ketamine on pain after arthroscopic rotator cuff repair: a prospective, doubleblind, randomized controlled study. Arch Orthop Trauma Surg 2016;136:233-239.

58. Kim DH, Lin Y, Beathe JC, et al. Superior trunk block: A phrenic-sparing alternative to the interscalene block: A randomized controlled trial. Anesthesiology 2019;131: 521-533.

59. Ko SH, Kang BS, Hwang CH. Ultrasonography- or electrophysiology-guided suprascapular nerve block in arthroscopic acromioplasty: a prospective, double-blind, parallel-group, randomized controlled study of efficacy. Arthroscopy 2013;29(5):794-801.

60. Koltka K, Koknel-Talu G, Asik M, Ozyalcin S. Comparison of efficacy of intraarticular application of magnesium, levobupivacaine and lornoxicam with placebo in 
arthroscopic surgery. Knee Surg Sports Traumatol Arthrosc 2011;19:1884-1889.

61. Kraeutler MJ, Reynolds KA, Long C, McCarty EC. Compressive cryotherapy versus ice-A prospective, randomized study on postoperative pain in patients undergoing arthroscopic rotator cuff repair or subacromial decompression. J Shoulder Elbow Surg 2015;24:854-859.

62. Lee H-J, Kim Y-S, Park I, Ha D-H, Lee J-H. Administration of analgesics after rotator cuff repair: A prospective clinical trial comparing glenohumeral, subacromial, and a combination of glenohumeral and subacromial injections. J Shoulder Elbow Surg 2015;24:663-668.

63. Lierz P, Losch H, Felleiter P. Evaluation of a single preoperative dose of etoricoxib for postoperative pain relief in therapeutic knee arthroscopy: A randomized trial. Acta Orthop 2012;83:642-647.

64. Lu J, Chen G, Zhou H, Zhou Q, Zhu Z, Wu C. Effect of parecoxib sodium pretreatment combined with dexmedetomidine on early postoperative cognitive dysfunction in elderly patients after shoulder arthroscopy: A randomized double blinded controlled trial. J Clin Anesth 2017;41:30-34.

65. Lynch JR, Okoroha KR, Lizzio V, Yu CC, Jildeh TR, Moutzouros V. Adductor canal block versus femoral nerve block for pain control after anterior cruciate ligament reconstruction: A prospective randomized trial. Am J Sports Med 2019;47:355-363.

66. Mahure SA, Rokito AS, Kwon YW. Transcutaneous electrical nerve stimulation for postoperative pain relief after arthroscopic rotator cuff repair: A prospective double-blinded randomized trial. J Shoulder Elbow Surg 2017;26:1508-1513.

67. Mardani-Kivi M, Karimi Mobarakeh M, Keyhani S, Haghighi M, Hashemi-Motlagh K, Saheb-Ekhtiari K. Arthroscopic bankart surgery: Does gabapentin reduce postoperative pain and opioid consumption? A tripleblinded randomized clinical trial. Orthop Traumatol Surg Res 2016;102:549-553.

68. Mardani-Kivi M, Karimi Mobarakeh M, Haghighi M, et al. Celecoxib as a pre-emptive analgesia after arthroscopic knee surgery: A triple-blinded randomized controlled trial. Arch Orthop Trauma Surg 2013;133:1561-1566.

69. Marinković D, Simin JM, Drasković B, Kvrgić IM, Pandurov M. Efficiency of ultrasound guided lower limb peripheral nerve blocks in perioperative pain management for knee arthroscopy in children. A randomized study. Med Pregl 2016;69:5-10.

70. McHardy PG, Singer O, Awad IT, et al. Comparison of the effects of perineural or intravenous dexamethasone on low volume interscalene brachial plexus block: a randomised equivalence trial. Br J Anaesth 2020;124:84-91.

71. Merivirta R, Äärimaa V, Aantaa R, et al. Postoperative fentanyl patch versus subacromial bupivacaine infusion in arthroscopic shoulder surgery. Arthroscopy 2013;29: 1129-1134.

72. Mitra S, Kaushal H, Gupta RK. Evaluation of analgesic efficacy of intra-articular bupivacaine, bupivacaine plus fentanyl, and bupivacaine plus tramadol after arthroscopic knee surgery. Arthroscopy 2011;27(12):1637-1643.

73. Moyano J, García M, Caicedo M. Analgesic Effect of Dexamethasone after Arthroscopic Knee Surgery: A
Randomized Controlled Trial. Pain Res Manag 2016;2016: 4216469.

74. Neuts A, Stessel B, Wouters PF, et al. Selective suprascapular and axillary nerve block versus interscalene plexus block for pain control after arthroscopic shoulder surgery: A noninferiority randomized parallel-controlled clinical trial. Reg Anesth Pain Med 2018;43:738-744.

75. Oh YN, Kim KN, Jeong MA, Kim DW, Kim JY, Ki HS. Effects of nefopam with fentanyl in intravenous patientcontrolled analgesia after arthroscopic orthopedic surgery: A prospective double-blind randomized trial. Turk J Med Sci 2018;48:142-149.

76. Sayın P, Dobrucalı H, Türk HŞ, Totoz T, Işıl CT, Hancı A. Effects of intra-articular levobupivacaine, fentanyl-levobupivacaine and tramadol-levobupivacaine for postoperative pain in arthroscopic knee surgery. Acta Orthop Traumatol Turc 2015;49(3):267-273.

77. Purcell RL, Brooks DI, Steelman TJ, et al. Fascia iliaca blockade with the addition of liposomal bupivacaine versus plain bupivacaine for perioperative pain management during hip arthroscopy: A double-blinded prospective randomized control trial. Arthroscopy 2019;35: 2608-2616.

78. Reda W, ElGuindy AMF, Zahry G, Faggal MS, Karim MA. Anterior cruciate ligament reconstruction: Is a tourniquet necessary? A randomized controlled trial. Knee Surg Sports Traumatol Arthrosc 2016;24:2948-2952.

79. Sanel S, Arpaz O, Unay K, Turkmen I, Simsek S, Ugutmen E. Comparison of intra-articular bupivacainemorphine with bupivacaine-tenoxicam combinations on post-operative analgesia in patients with arthroscopic meniscectomy: A prospective, randomised study. Int Orthop 2016;40:601-605.

80. Saritas TB, Borazan H, Okesli S, Yel M, Otelcioglu Ş. Is intra-articular magnesium effective for postoperative analgesia in arthroscopic shoulder surgery? Pain Res Manag 2015;20:35-38.

81. Schwartzberg RS, Reuss BL, Rust R. Efficacy of continuous subacromial bupivacaine infusion for pain control after arthroscopic rotator cuff repair. J Shoulder Elbow Surg 2013;22(10):1320-1324.

82. Shlaifer A, Sharfman ZT, Martin HD, et al. Preemptive analgesia in hip arthroscopy: A randomized controlled trial of preemptive periacetabular or intra-articular bupivacaine in addition to postoperative intra-articular bupivacaine. Arthroscopy 2017;33:118-124.

83. Spence D, Goff J, Mohan E, Bowen K, Osborne L, Maye J. Perioperative administration of gabapentin for shoulder arthroscopy: A prospective, randomized, double-blind, placebo-controlled study. AANA J 2011;79:S43-S50 (4 Suppl).

84. Syed UAM, Aleem AW, Wowkanech C, et al. Neer Award 2018: The effect of preoperative education on opioid consumption in patients undergoing arthroscopic rotator cuff repair: A prospective, randomized clinical trial. J Shoulder Elbow Surg 2018;27:962-967.

85. Tompkins M, Plante M, Monchik K, Fleming B, Fadale P. The use of a non-benzodiazepine hypnotic sleep-aid (Zolpidem) in patients undergoing ACL reconstruction: A randomized controlled clinical trial. Knee Surg Sports Traumatol Arthrosc 2011;19:787-791. 
86. Westergaard B, Jensen K, Lenz K, et al. A randomised controlled trial of ultrasound-guided blockade of the saphenous nerve and the posterior branch of the obturator nerve for postoperative analgesia after day-case knee arthroscopy. Anaesthesia 2014;69:1337-1344.

87. Wong AK, Keeney LG, Chen L, Williams R, Liu J, Elkassabany NM. Effect of local anesthetic concentration $(0.2 \%$ vs $0.1 \%$ ropivacaine) on pulmonary function, and analgesia after ultrasound-guided interscalene brachial plexus block: A randomized controlled study. Pain Med Malden Mass 2016;17:2397-2403.

88. Xing JG, Abdallah FW, Brull R, et al. Preoperative femoral nerve block for hip arthroscopy: A randomized, triplemasked controlled trial. Am J Sports Med 2015;43: 2680-2687.

89. Yun MJ, Oh JH, Yoon JP, Park SH, Hwang JW, Kil HY. Subacromial patient-controlled analgesia with ropivacaine provides effective pain control after arthroscopic rotator cuff repair. Knee Surg Sports Traumatol Arthrosc 2012;20:1971-1977.

90. Zhang Z, Zhu W, Zhu L, Du Y. Efficacy of celecoxib for pain management after arthroscopic surgery of hip: A prospective randomized placebo-controlled study. Eur J Orthop Surg Traumatol Orthop Traumatol 2014;24:919-923.

91. Zhou F, Du Y, Huang W, Shan J, Xu G. The efficacy and safety of early initiation of preoperative analgesia with celecoxib in patients underwent arthroscopic knee surgery: A randomized, controlled study. Medicine (Baltimore) 2017;96:e8234.

92. Thapa D, Ahuja V, Verma P, Gombar S, Gupta R, Dhiman D. Post-operative analgesia using intermittent vs. continuous adductor canal block technique: A randomized controlled trial. Acta Anaesthesiol Scand 2016;60: 1379-1385.

93. Lee Y-S, Park Y-C, Kim J-H, et al. Intrathecal hydromorphone added to hyperbaric bupivacaine for postoperative pain relief after knee arthroscopic surgery: A prospective, randomised, controlled trial. Eur J Anaesthesiol 2012;29:17-21.

94. Hartwell MJ, Selley RS, Terry MA, Tjong VK. Can we eliminate opioid medications for postoperative pain control? A prospective, surgeon-blinded, randomized controlled trial in knee arthroscopic surgery. Am J Sports Med 2020;48:2711-2717.

95. Davey MS, Hurley ET, Anil U, et al. Pain management strategies after anterior cruciate ligament reconstruction: A systematic review with network meta-analysis. Arthroscopy 2021;37:1290-1300.e6.

96. Wolff AB, Hogan GW, Capon JM, Napoli AM, Smith HJ, Gaspar PS. Pre-operative lumbar plexus block provides superior post-operative analgesia when compared with fascia iliaca block or general anesthesia alone in hip arthroscopy. J Hip Preserv Surg 2016;3:338-345.

97. YaDeau JT, Tedore T, Goytizolo EA, et al. Lumbar plexus blockade reduces pain after hip arthroscopy: A prospective randomized controlled trial. Anesth Analg 2012;115: 968-972.

98. Premkumar A, Samady H, Slone H, Hash R, Karas S, Xerogeanes J. Liposomal bupivacaine for pain control after anterior cruciate ligament reconstruction: A prospective, double-blinded, randomized, positive-controlled trial. Am J Sports Med 2016;44:1680-1686. 